\title{
In Vitro Studies of Neuronal Networks and Synaptic Plasticity in Invertebrates and in Mammals Using Multielectrode Arrays
}

\author{
Paolo Massobrio, ${ }^{1}$ Jacopo Tessadori, ${ }^{2}$ Michela Chiappalone, ${ }^{2}$ and Mirella Ghirardi ${ }^{3}$ \\ ${ }^{1}$ Neuroengineering and Bio-Nano Technology Lab (NBT), Department of Informatics, Bioengineering, Robotics and \\ System Engineering (DIBRIS), University of Genova, Via all'Opera Pia 13, 16145 Genova, Italy \\ ${ }^{2}$ Department of Neuroscience and Brain Technologies, Istituto Italiano di Tecnologia, Genova, Via Morego 30, 16163 Genova, Italy \\ 3“Rita Levi Montalcini” Department of Neuroscience, University of Torino, Corso Raffaello 30, 10125 Torino, Italy
}

Correspondence should be addressed to Mirella Ghirardi; mirella.ghirardi@unito.it

Received 31 December 2014; Accepted 27 February 2015

Academic Editor: George W. Huntley

Copyright (C) 2015 Paolo Massobrio et al. This is an open access article distributed under the Creative Commons Attribution License, which permits unrestricted use, distribution, and reproduction in any medium, provided the original work is properly cited.

Brain functions are strictly dependent on neural connections formed during development and modified during life. The cellular and molecular mechanisms underlying synaptogenesis and plastic changes involved in learning and memory have been analyzed in detail in simple animals such as invertebrates and in circuits of mammalian brains mainly by intracellular recordings of neuronal activity. In the last decades, the evolution of techniques such as microelectrode arrays (MEAs) that allow simultaneous, longlasting, noninvasive, extracellular recordings from a large number of neurons has proven very useful to study long-term processes in neuronal networks in vivo and in vitro. In this work, we start off by briefly reviewing the microelectrode array technology and the optimization of the coupling between neurons and microtransducers to detect subthreshold synaptic signals. Then, we report MEA studies of circuit formation and activity in invertebrate models such as Lymnaea, Aplysia, and Helix. In the following sections, we analyze plasticity and connectivity in cultures of mammalian dissociated neurons, focusing on spontaneous activity and electrical stimulation. We conclude by discussing plasticity in closed-loop experiments.

\section{Introduction}

Communication among neurons is essential for higher brain functions, such as perception, memory, and movement. The mature nervous system is an intricate network in which neurons are extensively interconnected to each other. These connections are made up during embryonic and postnatal development and are modified during life by experience. As suggested by early experiments of Sperry [1-3], during circuit development axon-target recognition relies on chemical matching. Intercellular signals such as adhesion molecules, chemoattractants, and neurotrophic factors, remarkably well conserved during evolution, provide crucial directions to the developing nervous system [4-6]. In addition, electrical activity in the forming circuits also plays a critical role in shaping connectivity, as shown by the pioneering experiments of Hubel and Wiesel in the development of the visual cortex [7-11].
Activity-dependent fine-tuning of neuronal circuitry is not limited to early development and neural circuits are adaptable even in the mature individual [12]. As firstly proposed by Hebb in the 1940s, synaptic strength increases when the pre- and postsynaptic elements are synchronously active [13]. The existence of synapses whose efficiency is remarkably influenced by previous activity and that undergo long-term potentiation (LTP) has been discovered in rabbit hippocampus by Bliss and Lomo [14] and then long-term depression (LTD) has been observed in the cerebellar cortex [15].

Synaptic plasticity has long been implicated in cognitive processes such as learning and memory [16-18]. Interestingly, the same processes of plasticity described in simple nervous systems of invertebrates such as Aplysia, Helix, Lymnaea, Helisoma, and Drosophila seem to be conserved in mammals and many similar molecular mechanisms underlining both simple and complex forms of learning and memory $[16,17]$. 
In the last decades, cellular and molecular aspects of synaptic plasticity have been analyzed in detail in circuits of few cells, while less information is currently available about dynamics of large populations of neurons during development and plastic modifications. Experimental analysis of connectivity with simultaneous multiple site recordings within functional neuronal networks is therefore a promising approach in neuroscience research.

Nowadays, microelectrodes arrays (MEAs) are the state of the art for studies on neuronal network dynamics. By means of those devices it is possible to characterize the neuronal dynamics of several biological preparations (i.e., from invertebrates $[21,22]$ to different cerebral mammalian areas such as cortex [23] and hippocampus [24]) by studying their development [25] and delivering electrical [26-28] or chemical stimulation [29-31] to induce synaptic plasticity at the network level [20]. Recently, improvements to the MEA technology have led to an increase in the number of recording sites [3236]. In this way, the idea to have one neuron plated over the surface of each electrode (1:1 coupling) becomes achievable, thus implying the possibility of realizing neuronal networks with a precise and well identified topology. Signal recording systems (microtransducers) based on MEAs and field effect transistors (FETs) have been established as powerful tools for recording the electrical activity of networks of neurons cultured in vitro [30,37-41]. Under this experimental condition, neurons are directly connected to the microtransducers by a neuroelectronic junction, and the neuronal electrical activity is noninvasively extracellularly recorded over long periods of time.

\section{Microtransducer Array Technologies}

2.1. Passive Microtransducers. MEAs are made of cell-sized electrodes (10-100 $\mu \mathrm{m}$ diameter) placed onto a glass or on a printed circuit board substrate. The electrodes, typically made of gold, indium tin oxide (ITO), titanium nitride (TiN), or black platinum, are biocompatible, long-lasting, and preferably with a low impedance (less than $500 \mathrm{~K} \Omega$ at $1 \mathrm{kHz}$ ) for low thermal noise. The MEA surface and electrode leads are coated with biocompatible insulators (e.g., polyamide or silicon nitride/oxide) which prevent short circuits with the electrolyte bath. These insulators, coated with adhesion molecules such as polylysine and laminin, allow and help neuronal coupling to the device surface. Finally, the low impedance of the electrodes and the choice of a correct voltage range for avoiding the generation of neurotoxic redox complexes allow the delivery of external stimuli.

The fabrication of MEAs is based on the thin-film technology [42]. MEAs can be grouped on the basis of the number of electrodes which define the array (from 60 to 10,000 electrodes), the electrode size (from 10 up to $30 \mu \mathrm{m}$ ), and the interelectrode spacing (from 20 up to $500 \mu \mathrm{m}$ ). Most of the results presented in this review are based on MEAs with 60 flat round electrodes made of titanium nitride. Tracks and contact pads are made of titanium or ITO, and the insulation material consists of silicon nitride $\left(\mathrm{Si}_{3} \mathrm{~N}_{4}\right)$. The electrodes are positioned in an $8 \times 8$ layout grid with missing corner electrodes. ITO contact pads and tracks are transparent to allow a perfect view of the specimen under the microscope. Finally, a glass ring is placed at the center of the array in order to contain the culture medium. In this way the cultures may survive for several weeks when placed in an incubator.

2.2. FET-Based Microtransducers. A considerable contribution in the microtransducer field for electrophysiological neuronal activity recording was made by Fromherz and coworkers [37, 40, 43-46]. They demonstrated that opengate FETs are able to detect the transient extracellular voltage beneath a single neuron attached, through its cell membrane, to the gate insulator of a FET. Briefly, a field-effect transistor consists of four terminals (Figure 1(a)): the source (S), the drain (D), the gate $(G)$, and the bulk (B). The region between the source and drain defines the channel. The gate is separated from the channel by an insulator $\left(\mathrm{SiO}_{2}\right.$ or $\left.\mathrm{Si}_{3} \mathrm{~N}_{4}\right)$. The source and drain connections are degenerately doped $n$-type silicon, while the bulk of the transistor is p-type. If there is any kind of voltage applied to the gate, the source and drain are electrically disconnected. If a positive voltage is applied to the gate, positive charges in the gate will electrostatically repel the holes in the underlying $p$-type channel. If the applied bias is further increased, the positive charges on the gate will attract minority carriers (electrons) and a channel that will link the source and the drain together will take place, allowing the passage of current. Thus, one can modulate the drain-source current by applying a particular gate voltage. For the use of a FET as microtransducer to record electrophysiological activity, the metallic gate is removed. In this way, the insulator is directly exposed to the cell membrane and to the electrolytic solution. The activity of the neuron leads to ionic and displacement currents flowing through the attached membrane, resulting into an extracellular voltage drop along the narrow cleft between the membrane and the gate insulator. The change of the extracellular voltage induced by the neuronal activity gives rise to an electric field across the insulator which modulates the drain-to-source current of the FET; this current, transduced into a voltage, describes the extracellular recorded signal probed by the microtransducer. Compared to the first generation of FET-based systems [37, 46], nowadays low-noise level FET-based systems are available. The introduction of low-noise transistors [47] permits recording the electrophysiological activity also from mammalian neurons (cortical or hippocampal neurons) which exhibit a peak-topeak amplitude smaller $(\sim 100 \mu \mathrm{V})$ than the one originated by large invertebrate neurons (up to tens of millivolts). We can then conclude that, with respect to conventional MEAs, three main advantages can be achieved by using this kind of technology: (i) heavy parallelization-large numbers of microtransducers can be addressed by on-chip multiplexing architectures. In this way, it becomes feasible to design array of microtransducers with thousands of recording sites. This kind of devices can be exploited to study the emerging connectivity in large-scale neuronal networks; (ii) signal quality - the signal is conditioned right at the transducer by means of dedicated circuitry units; (iii) ease of handling and use-both devices and signals are robust.

2.3. Increasing the Coupling between Neurons and Microtransducers. In the last decades, several attempts have been made 


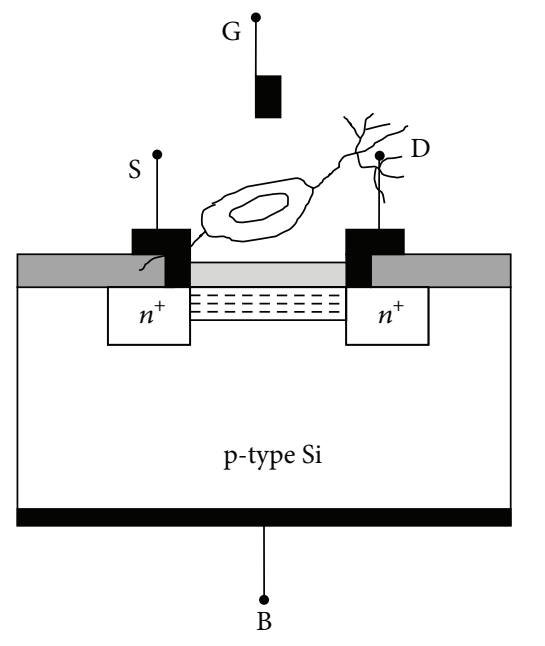

Metallization

Insulator

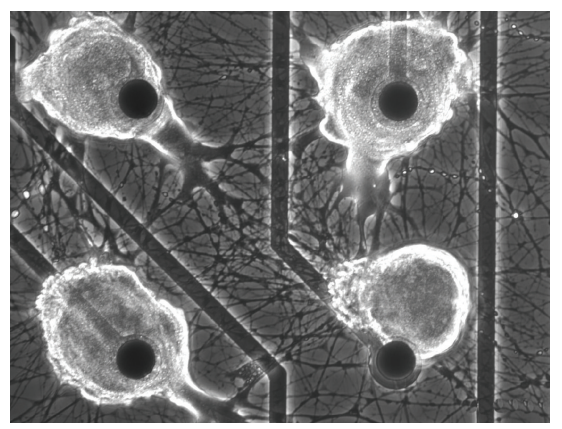

$\overline{50 \mu \mathrm{m}}$

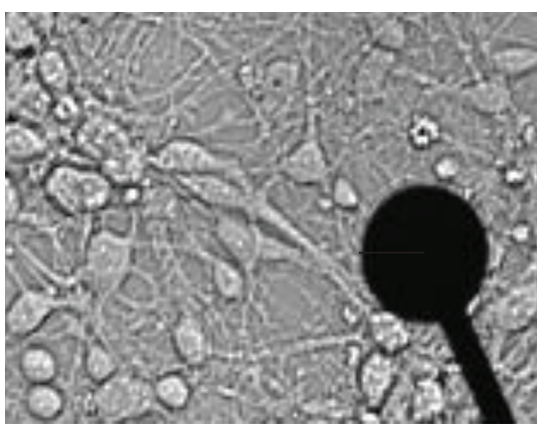

$\overline{15 \mu \mathrm{m}}$
E=ョ Channel

(a) (b) (c)

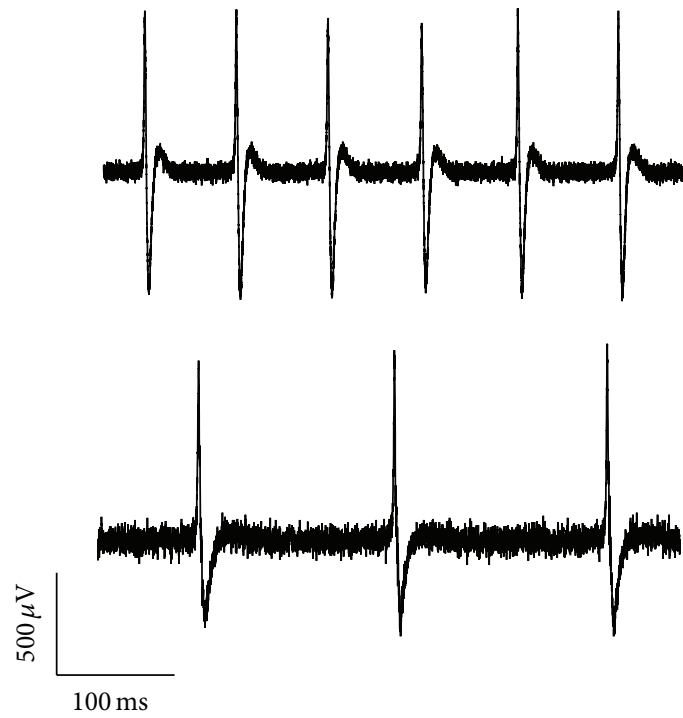

(d)

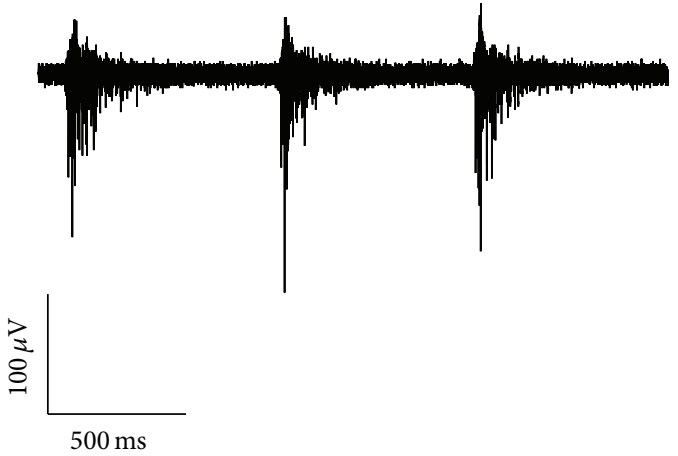

(e)

FIGURE 1: (a) Schematic representation of a field-effect transistor (FET). (b) Example of Helix neurons 24 hours after plating on MEA. Scale bar is $50 \mu \mathrm{m}$. (c) Cortical neurons after 24 days in vitro coupled to a MEA. Scale bar is $15 \mu \mathrm{m}$. (d) Extracellular action potentials recorded by a MEA relative to B2 (top) and C1 (bottom) Helix neurons. (e) Typical bursting activity of a cortical network recorded by a MEA.

to improve the coupling between neurons and the surface of the microtransducers with the aim of increasing the amplitude of the recorded signals and achieving more stable adhesion conditions. In this sense, a significant result has been obtained by the use of carbon nanotubes (CNTs), considered a promising material for the assembly of nanodevices. Recent studies have suggested the great potential of high-density $\mathrm{CNT}$ coated surfaces as an interfacing material with neuronal systems; moreover, $\mathrm{CNT}$ surfaces act as an extremely efficient biocompatible substrate on which neurons adhere and proliferate [48-51]. Furthermore, the CNT-based neuroelectronic junction [52] plays a relevant role on the extracellular neuronal signal recording [53] and stimulation [54].

More recently, a great step towards the possibility to enhance the coupling between electrogenic cells and microtransducers has been made by Spira and coworkers who developed an innovative extracellular recording technique, based on mushroom-shaped protruding microelectrodes [55, 56]. This extracellular system allows in-cell recording not only of action potentials, but also of subthreshold synaptic inputs from individual neurons with a signal-to-noise ratio that matches that of conventional intracellular recordings. 


\section{Invertebrate Neurons and MEA Microchips}

Invertebrate neurons have represented an important tool in the development of microtechnologies applied to neurosciences. Specifically, invertebrate neurons have a large cell body which facilitates the formation of high quality neuron/microtransducer interfaces; they are easily identifiably amid the ganglia and may grow in isolated culture as well as in reconstructed specific circuits. In addition, invertebrates neural circuitry is very simple when anatomically compared to mammals, but it exhibits many types of short and long-term plasticity that have been extensively studied at the behavioral, cellular, and molecular levels $[17,57]$. Moreover, by exploiting the size of their somata and the amplitude of their action potentials, invertebrate neurons have also been used as testbed for innovative microtransducers. In 1991, a Retzius cell of the leech Hirudo medicinalis was successfully coupled for the first time to a transistor by Fromherz and colleagues [37, 58], and bioelectrical signals were elicited from neurons of the pond snail Lymnaea stagnalis grown on microchips and connected by gap junctions $[44,45,59-61]$.

3.1. Chemical Connections and Plasticity in Lymnaea Neurons. A further important step has been the reconstruction on the silicon chip of a chemical synapse of Lymnaea that exhibited plastic properties [62]. In Lymnaea, the respiratory neuron VD4 (visceral dorsal 4) forms a cholinergic synapse with the neuron LPeD1 (left pedal dorsal 1) $[63,64]$ and this connection can be reconstituted in culture in a somasoma configuration [64-68]. The VD4-LPeD1 synapse undergoes short-term plastic changes and intracellular tetanic stimulation of VD4 induces an enhancement of synaptic transmission in the postsynaptic neuron LPeD1 $[69,70]$. Interestingly, similar potentiation was obtained when the presynaptic neuron VD4 was repeatedly stimulated by a chip capacitor and postsynaptic excitation in LPeD1 was recorded by a transistor, establishing for the first time a short-term plasticity in a pair of chemically connected identified cells cultured on the electronic chip [62]. This result provided evidence that interfacing semiconductor chips and neurons may represent a direct noninvasive method for short- and long-term studies of plasticity in vitro.

Simultaneous recordings from a large number of neurons of central pattern generator (CPG) networks that mediate fundamental Lymnaea behaviors including feeding and respiration [71-74] have been obtained from semi-intact preparations consisting of ganglia and sensory input interfaced to MEAs [75]. In this preparation the MEA was used to monitor learning-induced changes in the electrical responses of specific types of identified buccal feeding motoneurons [76-83]. Using a protocol for in vitro single-trial classical conditioning of Lymnaea feeding behavior [84], plastic changes were induced in the semi-intact preparation. Recordings with MEA technology have allowed detecting modifications in the spatiotemporal firing patterns of a large number of feeding neurons. This proved that the feeding CPG of the pond snail is associated with another oscillating neuronal population [85] characterized by activity alternating with quiescence during which the CPG is refractory to activation by food-associated stimuli. Similar network refractory periods have previously been observed in the rhythmic activity networks of spinal interneurons from locomotor CPG regions in culture as well as in the spinal cord of embryonic rat $[86,87]$ and chick [88]. In Lymnaea circuits interfaced with MEA, the dynamic of network refractory periods of CPG was modulated by dopamine [85], a neurotransmitter known to regulate feeding behavior and reward [89].

3.2. Multisite Detection of Dopamine Release from Lymnaea Neurons. Recently multifunctional MEAs were developed for neuroelectrical and neurochemical recordings in vivo $[90,91]$ and in vitro that allowed the detection of neurotransmitters such as dopamine released from neurons of acute hippocampal slices [92] and from PC12 cell lines [93]. Neurotransmitters released from presynaptic terminals regulate neuronal communications and it is well known that alteration in electrical activity and in level of neurotransmitters underlies several disorders such as Parkinson's disease, schizophrenia, and major depression [94, 95]. Multimodal probes for simultaneous activity and chemical detection appear suitable for analyzing dynamics of activity correlated to neurochemical release in complex circuits and for investigating the effects of drugs employed in treatments of neurological diseases.

More details on the specific sites of neurotransmitter release from a neuron have been acquired with a planar microelectrode array that has been fabricated and characterized by Patel et al. [96] for simultaneous multisite detection of dopamine release. Electrically evoked dopamine release was observed from freshly isolated dopaminergic RPeD1 neurons of Lymnaea stagnalis. These large neurons were plated on MEAs containing electrodes spatially arranged to allow the simultaneous recordings from various structural regions of an isolated cell. Evoked recordings of dopamine release induced by tetanic stimulation were obtained simultaneously from distinct locations such as the soma, the axon, and the terminals. Interestingly, repeated recordings at various timepoints showed that the release of neurotransmitter varied over the structural regions of the Lymnaea neuron during the reorganization of the cell following isolation from the ganglia. MEA recordings of simultaneous spatiotemporal responses of dopamine released by action potential activation from cultured large neurons could be used to study the changes in neurotransmitter release in specific regions of the neurons during formation of synapses and neuronal network activity.

3.3. "In-Cell" Recording in Aplysia Neurons. Many extracellular electrodes such as noninvasive extracellular MEAs for in vitro recordings can reliably measure action potentials but subthreshold potentials such as synaptic potentials remain undetectable. A large number of events in synaptic plasticity are associated with changes in amplitude of synaptic potentials that very often do not reach the threshold required for spike firing [16]. In the last decade, many attempts have been performed to improve the electrical coupling between cultured cells and planar MEAs and to reduce the junctional membrane resistance. A novel neuron-electrode configuration ("in cell" recording) developed by the group of 
Spira [55, 56, 97-100] allowed recording action potentials as well as synaptic potentials with MEAs in Aplysia neurons: it consists of an array of noninvasive gold, mushroom-shaped microelectrodes that permits simultaneous, multisite, longterm recordings of action potentials and subthreshold potentials with quality and signal-to-noise ratio comparable to that obtained with intracellular sharp glass microelectrodes or patch electrodes.

Neurons from the buccal and abdominal ganglia of Aplysia californica were isolated with their initial axon and maintained in culture [101, 102]. Plated Aplysia neurons survive in culture for over a month, extending neurites and forming chemical and electrical synapses [103]. Isolated Aplysia neurons were manually plated directly on top of the microelectrodes that protrude from the glass substrate with three-dimensional mushroom geometry and dimensions that mimic dendritic spines in their shape and sizes. A peptide that induces phagocytotic activity was covalently linked to the microelectrodes in order to generate efficient contact between them and the neurons. Electron microscopy showed that the neuron-microelectrode has a reduced cleft width with increased contact area [56] and within a few minutes of contact there was a restructuring of the actin cytoskeleton to form an actin ring around the stalk of the microelectrode that became a stable cytoskeleton structure and was maintained for several days as shown by live confocal microscopy [99]. Ultrastructural observations revealed that other cell types such as $\mathrm{CHO}$, embryonic fibroblast cells NIH/3T3, rat myocardium cells $\mathrm{H} 9 \mathrm{C} 2$, and rat adrenal medulla $\mathrm{PC} 12$ cell lines engulf the head and stalk of the gold spines [56]. The goldspine matrix influences the growth of neurons but they maintain typical electrophysiological properties and form functioning synapses. These electrodes represent an improved substrate for the assembly of neuroelectronic devices well suited for the study of neuronal plasticity as they allow the detection of subthreshold potentials in Aplysia neurons.

3.4. Helix Circuits: Morphological and Electrical Development. Signals from single or paired invertebrate neurons have been recorded with MEA devices for many years but the first long-lasting study on invertebrate neuronal networks in culture was performed in 2013 by Massobrio and colleagues [19]. They characterized the dynamics and connectivity of networks made up of neurons of the land snail Helix aspersa coupled to MEAs during their development for several days. Previous experiments showed that C1, C3, and B2 neurons from buccal and cerebral ganglia of Helix form microcircuits in vitro $[22,104-107]$ and the serotonergic connection C1B2 involved in the regulation of feeding behaviors of Helix snails reconstructed in culture undergoes plastic changes [105]. The large size of Helix neurons (soma diameter up to $100-150 \mu \mathrm{m}$; see Figure 1(b)) compared to mammalian neurons (see Figure 1(b)) permits a 1:1 coupling between neurons and microelectrodes, facilitating the study of the relationships between the electrophysiological activity of individual neurons in a network and their development of neurite outgrowth and connections in the long term.

Helix neurons start to develop synaptic connections a few hours after plating, and their development is faster than cortical cultures from mammals [25]. Figure 1 shows the growth of Helix neurons that have been plated on top of the microelectrodes 24 hours earlier (1B) and of cortical neurons after 24 days in culture (1C).

A peculiarity of these invertebrate neuronal networks is the absence of spontaneous activity: Helix neurons are generally silent on MEAs [19, 22, 108, 109], and spontaneous firing was observed only occasionally [22]. Figure $1(\mathrm{~d})$ shows two examples of extracellularly recorded signals coming from stimulated B2 (top) and C1 (bottom) neurons. Typically, the signals from these neurons display amplitudes greater than $500 \mu \mathrm{V}$, differently from mammalian spikes that, although spontaneously active, seldom are greater than $200 \mu \mathrm{V}$. Figure 1(e) shows a typical cortical bursting sequence. Therefore, to study Helix networks, neuronal activity was triggered by means of chemical treatments that induce general depolarization of the cell membrane potential (potassium chloride, $\mathrm{KCl}$, Figure 2(a)) or that selectively depolarize B2 neurons (serotonin, 5-HT, Figure 2(b)). Thus, the neuritic outgrowth and the induced activity were followed during development of the circuits for several days, as shown in Figure 2. The firstorder statistics were used to characterize neuronal dynamics such as interspike interval (ISI) and firing rate. The crosscorrelation function [110] was employed to estimate the functional connections established among neurons of the network in order to reconstruct the topological connections and monitor them during development. The simplicity of these neuronal circuits allows the achievement of a good matching between morphological and functional links on MEA. It has been found that both chemical stimulations were efficacious in triggering firing activity in Helix circuits, but a long-lasting change in activity occurred only with 5-HT treatment, as presented in Figure 2. For as long as the network evolved, an increase with time of functional connections was detected. Moreover, the analysis of spiking activity as well as their functional linked latencies showed that networks treated with 5-HT displayed a dynamic modulated mostly by chemical synapses, while a predominance of electrical connections occurred in $\mathrm{KCl}$-triggered networks.

The preferential formation and strength of chemical or electrical synapses during circuit development are critically regulated by several factors including neuromodulators such as serotonin and dopamine, as recently reviewed by Pereda [111]. The prevalence of chemical connections in 5-HTtreated circuits may underline the long-term maintenance of spontaneous activity. Serotonin applications trigger activity through the depolarization of B2 neurons and the increased excitability of $\mathrm{C} 1$ neurons, and continuous release of 5HT from the firing neurons may maintain it, as previously reported [112-114]. In addition, it has been demonstrated that 5-HT selectively prevents the formation of electrical synapses while allowing chemical synaptogenesis between identified Helisoma trivolvis neurons $[115,116]$, maybe due to a negative modulation of neurite elongation [117-121] and a direct action on gap junctions [122-124], also observed in neocortical circuits [125]. In mammals, 5-HT has been implicated in shaping neuronal connectivity by decreasing neurite branching in rat cortical neurons during development [126] and impairing neurite density in mouse organotypic 

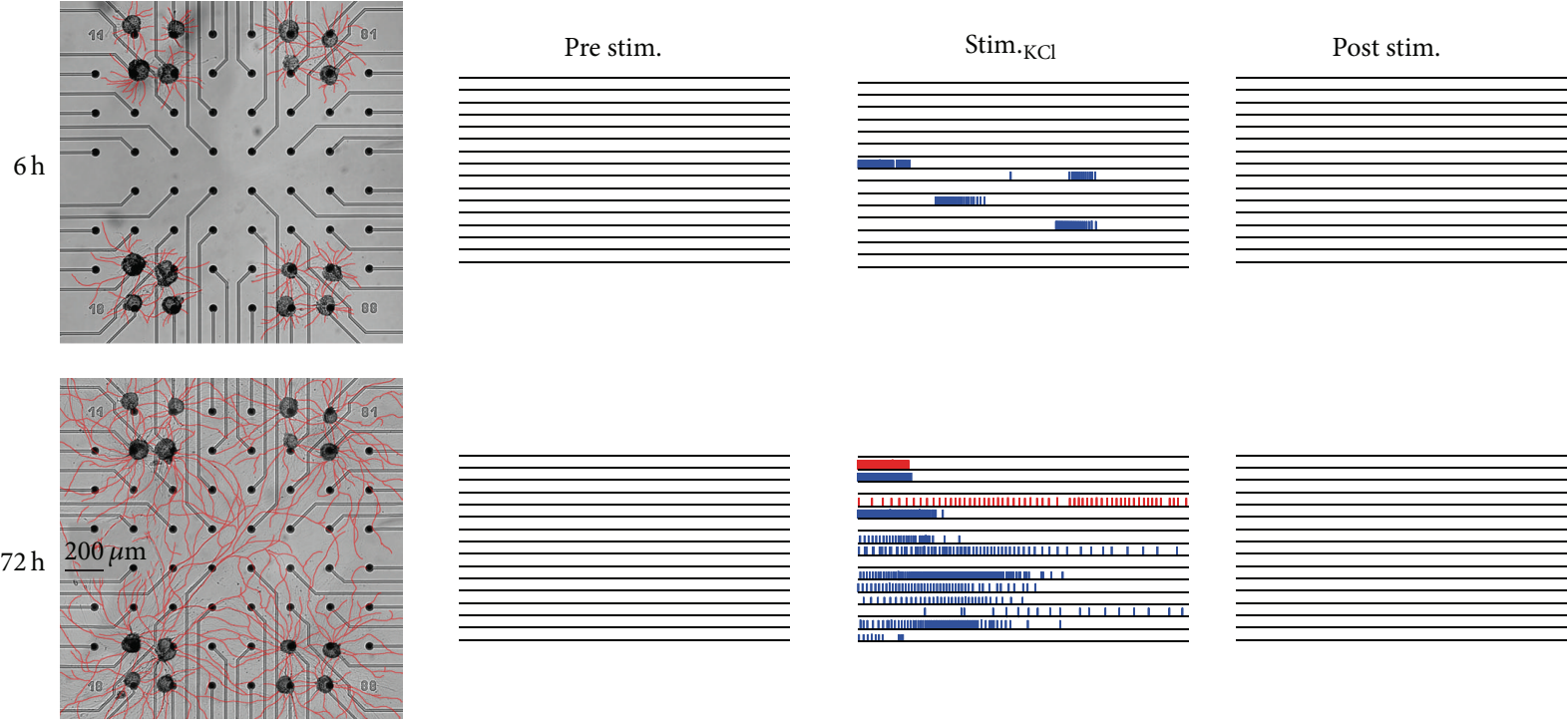

(a)
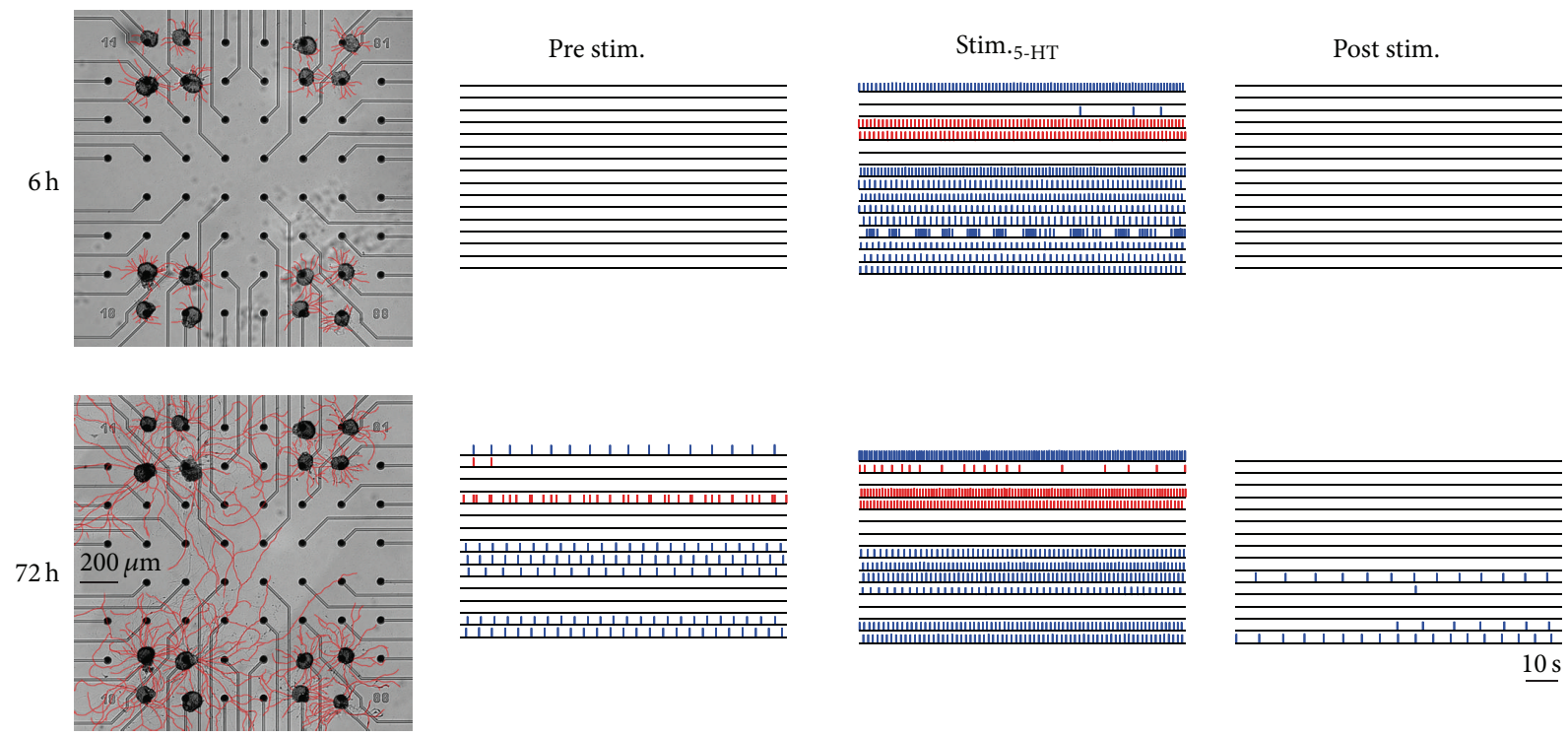

(b)

FIGURE 2: Example of Helix cultures treated with $\mathrm{KCl}$ (a) or 5-HT (b) applications. Each block represents a time-point of recordings (i.e., 6, 72 hours after plating). In the first column, the development of the neurite arborizations is indicated (scale bar is $200 \mu \mathrm{m}$ ). The three columns of raster plots show one minute of electrophysiological activity just before, during, and after the $\mathrm{KCl}$ or 5-HT treatment. Red and blue lines indicate recordings from $\mathrm{C} 1$ and $\mathrm{B} 2$ neurons, respectively. Scale bar is $10 \mathrm{~s}$. Modified from [19].

slice cultures [127]. The increase in neurite density induced by $\mathrm{KCl}$ treatments may contribute to enhancing cell-cell contact, thus promoting a higher coupling coefficient among cells [118]. Since gap junctions likely play a fundamental role in determining network synchronization $[128,129]$, signals may reverberate among neurons until they return to a silent state by switching off the circuit.

Following repeated stimulations, similar dynamics during the development of these invertebrate circuits were observed: network activity soon reached values of firing rates and ISIs which remained almost unchanged during the development despite connectivity maturation. This behavior is very distinctive, especially if compared with studies regarding the development of in vitro cortical neurons from rat embryos [25]. Indeed cortical assemblies were found to change their electrophysiological patterns as a function of network maturation, probably associated with a much higher synapse density $[130,131]$.

\section{Plasticity and Connectivity in Mammals}

4.1. Mammalian Neuronal Assemblies Display Spontaneous Electrophysiological Activity. Cultured neurons extracted from both embryonic and postnatal rodents are spontaneously active. This activity, originating from the interactions of 
TABLE 1: Summary of the plasticity protocols used in mammalian neuronal cultures.

\begin{tabular}{|c|c|c|}
\hline Reference & Year & Plasticity protocol \\
\hline Maeda et al. [139] & 1998 & $\begin{array}{l}20 \text { bursts at } 0.2 \mathrm{~Hz} \text {, each with } 11 \text { pulses at an intraburst frequency of } 20 \mathrm{~Hz} \text { delivered } \\
\text { from } 5 \text { electrodes }\end{array}$ \\
\hline Jimbo et al. [140] & 1998 & 11 bursts at $0.2 \mathrm{~Hz}$, each with 11 pulses at an intraburst frequency of $20 \mathrm{~Hz}$ \\
\hline Jimbo et al. [141] & 1999 & 10 bursts at $0.2 \mathrm{~Hz}$, each with 11 pulses at an intraburst frequency of $20 \mathrm{~Hz}$ \\
\hline Tateno and Jimbo [142] & 1999 & $\begin{array}{l}10 \text { bursts at } 0.2 \mathrm{~Hz} \text {, each with } 11 \text { pulses at an intraburst frequency of } 20 \mathrm{~Hz} \text {, also } \\
\text { delivered from a pair of electrodes }\end{array}$ \\
\hline Shahaf and Marom [143] & 2001 & $\begin{array}{l}\text { Adaptive stimulation between a pair of electrodes at } 1-3 \text { s intervals repeated until } \\
\text { the desired response is achieved (or for } 10 \min \max \text { ) }\end{array}$ \\
\hline Ruaro et al. [144] & 2005 & $\begin{array}{l}\text { Bursts of } 100 \text { pulses delivered at the frequency of } 250 \mathrm{~Hz} \text { from } 15 \text { electrodes } \\
\text { recreating an "L-shape" }\end{array}$ \\
\hline Wagenaar et al. $[25,145]$ & 2006 & $\begin{array}{l}150 \text { trains of } 20 \text { pulse pairs. Bursts suppressed by means of a high frequency }(50 \mathrm{~Hz}) \\
\text { distributed stimulation (not during tetanus delivery) }\end{array}$ \\
\hline Chiappalone et al. [20] & 2008 & $\begin{array}{l}\text { Jimbo protocol with additional trains of pulses at } 0.2 \mathrm{~Hz} \text { falling in the middle of the } \\
\text { tetanic burst }\end{array}$ \\
\hline Le Feber et al. [146] & 2010 & Slow electrical stimulation $(0.2-0.33 \mathrm{~Hz})$ \\
\hline
\end{tabular}

neuronal assemblies, is a peculiar feature of the mammalian nervous system and it can be found at different levels of investigation: in the cerebral cortex, it takes the shape of oscillatory patterns which span over different rhythms [132]; in reduced in vitro models, like dissociated cortical cultures, spontaneous activity is mainly characterized by a mixture of spikes and bursts lasting from a few to hundreds of milliseconds [38]. Such dynamic evolves, since it changes as a function of the degree of development of the cultures [133]: during the first stages of development, dissociated cortical assemblies display mainly irregular and asynchronous spiking activity; from the second week in vitro, spikes tend to cluster into bursts, a signature feature that persists throughout the time in culture, thus representing the mature state of the network. Those bursts can be found in both hippocampal [24] and cortical cultures [134] and are similar to activity patterns of in vivo systems deprived of afferent stimuli or with pathologies like epilepsy [135]. On the other hand, Eytan and Marom [136] noted similarities between the dynamics of these in vitro "synchronized bursting events" and population responses recorded in vivo while animals are engaged in sensory, motor, or "internal" cognitive tasks.

The presence of this "cumbersome" ongoing activity, exhibiting a high degree of variability, makes it difficult to interact with these cortical ensembles. However, several attempts to modulate these irregular dynamics by means of appropriate electrical and/or chemical stimulations can be found in the literature. In general, low-frequency, uniform, sustained electrical stimulation locks the phase of periodic bursts to the applied stimuli [137]. Higher rates of stimulation induce a transition from synchronized bursting activity into a sparse spiking behavior, more similar to in vivo awake cortical dynamics [27]. Conversely, an electrical stimulation pattern tailored on the network endogenous activity is able to efficiently induce modifications in the network synchronization and, in particular, it affects the network bursting properties, by increasing both firing and bursting rate [138]. Moreover, after this kind of spontaneous activity-tailored stimulation, the strongest connections respond by further increasing their strength relative to other connections within the network. This mechanism likely preserves connections that are more informative and relevant to the overall network activity.

\subsection{Searching for Plasticity with MEAs: A History 20 Years} Long. The history of MEA studies demonstrating functional plasticity in cultured mammalian networks began in the 1990s with the pioneering work of Maeda and coworkers who reported that tetanic stimulation through one or more electrodes was able to induce plasticity [139]. In their work, they found that the probability of evoking bursts by test pulses, as well as a change in the rate of spontaneous bursting, was modified after the delivery of a strong tetanic stimulation. Less than one year later, Jimbo and coworkers observed similar results with a milder tetanic stimulation [140]. Following those experiments, numerous other attempts have been performed by several MEA labs worldwide aimed at finding whether peculiar features of the electrical stimulation (e.g., frequency, number of stimulated electrodes, and amplitude of the stimuli) were able to induce synaptic changes in the dynamics of the networks. Table 1 summarizes the most significant plasticity studies.

However, despite the different attempts pursued during these years, a clear answer to the question of whether neuronal cultures can learn thanks to plasticity phenomena is still controversial [147], mainly because of two reasons: the first is the difficulty of designing a network stimulation protocol (partly linked to the nonstationary behavior of the dissociated cultures) capable of reliably inducing changes, as we will report in the next sections. The second reason lies in the lack of an "electrophysiological endpoint" that can be easily correlated to plasticity in dissociated networks. Regarding the latter, most scientists supported the hypothesis of Marder and Buonomano [148]. They proposed that not only changes in the synaptic potentials but also changes in the firing patterns of neurons should be taken into account in long-term forms of plasticity. Moreover, the neuronal input/output function of the entire network must be studied and characterized to better understand the computational effects of plasticity on 
a long-term perspective [148]. According to this approach, MEA could become the gold-standard tool to define longterm network plasticity (LTNP) experiments [20]. Similarly, in 2006 Potter's lab coined the expression functional plasticity (FP) to indicate "those changes in stimulus-response relationships or in spontaneous patterns that are experimentally induced by electrical stimulation and lasting at least on the order of one hour" [145]. Phenomena like LTP and LTD fall within the boundary of this definition (and can thus be considered examples of functional plasticity), whereas short-lasting changes such as paired pulse facilitation and depression do not (their duration is much shorter than the one-hour limit).

In 2006, Potter's group published an interesting paper in Journal of Negative Results in Biomedicine. The authors applied different protocols to induce plasticity to a large set of cortical cultures coupled to MEAs [145]. The conclusions of their work are straightforward: bursting suppression obtained through distributed electrical stimulation is a prerequisite for inducing plasticity [27]. Their protocol consisted in the random selection of electrodes in the pool of those evoking electrical responses, followed by stimuli delivery in cyclic order, with an interstimulus interval of $20 \mathrm{~ms}$. This resulted in a complete, but reversible, cessation of spontaneous bursting. Only after this was achieved, the application of a tetanic stimulation resulted in plasticity in their experiments.

The works of other groups determined the fact that complete bursting suppression is not a strict prerequisite and, through appropriate experimental precautions, different tetanic stimulation protocols resulted in significant connectivity changes even in the presence of spontaneous bursting. Several of those papers are discussed in more detail in the following chapters.

4.3. Stable Recordings Are Necessary to Induce Plasticity. Stability and responsiveness of culture batches are the first and most important conditions to be assessed to induce plasticity. As stated in the previous sections, both cortical and hippocampal assemblies display a mixture of spiking and bursting activity, with a high degree of variability (e.g., percentage of random spiking activity and frequency of the bursting activity) from culture to culture. For this reason, Chiappalone and coworkers developed a procedure that evaluates the level of nonstationarity of the spontaneous and stimulusevoked activity of a neuronal network [20]. The application of this criterion allows discarding of cultures displaying large variations of dynamics which can obscure changes in the synaptic efficacy induced by plasticity protocols. Cultures are selected on the basis of their initial spontaneous activity and on their ability to respond to low-frequency stimulation. In particular, two conditions regarding the spontaneous activity and two conditions regarding the stimulus-evoked activity should be met. Firstly, the initial value of firing rate should be above a defined threshold (set at 3 in [20]), and secondly the firing rate should remain stable between the phases of spontaneous activity before the plasticity protocol delivery. As for stimulus-evoked activity, a stimulated electrode is not considered for future stimulations if it is not able to evoke a global response in at least $50 \%$ of the recording electrodes. Finally, the network response to low-frequency (i.e., $0.2 \mathrm{~Hz}$ ) "test" stimuli has to be stable; that is, the variation of the evoked response, evaluated by means of the peristimulus time histogram (PSTH), has to be below a defined threshold. Figure 3(a) shows a bilogarithmic plot of the mean spontaneous firing rates. Spontaneous activity of each network was measured at the beginning of the experiment $\left(\mathrm{MFR}_{\text {pre }}\right)$ and after the first test stimulus session $\left(M R_{\text {post }}\right)$. Curved black lines define the confidence intervals (see [20]). Cultures labeled with red circles verify the MFR stability conditions and are potentially able to show plastic behavior. On the other hand, blue circles indicate cultures with a low firing rate, while yellow and green circles represent unstable cultures exhibiting spontaneous increase (yellow) or decrease (green) of their firing rate. Figure 3(b) shows an example of a PSTH map. The two superimposed profiles of the PSTH represent the probability of response ( $y$-axis: $[0,1])$ of the network to two different sessions of stimulation (black and red) in a time window of $400 \mathrm{~ms}$ ( $x$-axis: [0, $400 \mathrm{~ms}]$ ). We can observe that (i) all the electrodes are responsive and (ii) the two traces are practically superimposed, thus indicating stability of the preparation.

4.4. Two Families of Plasticity Protocols: Slow versus Fast Electrical Stimulation. The protocols currently used to induce plasticity both in vitro $[14,149]$ and in vivo $[150,151]$ consist of a massively synchronous, high-frequency stimulation (40$100 \mathrm{~Hz}$ ) named tetanic stimulation. Although these patterns of stimulation are extremely efficient, they have the drawback to rarely occur in nature [152]. The pioneering works which made use of tetanic stimulation also in cortical cultures coupled to MEAs were carried out in the groups of Maeda [139] and Jimbo [140]. Maeda and coworkers found that tetanic stimulation delivered by means of one or several electrodes induced plasticity [139]. They observed a change in the probability of evoking bursts by test pulses, as well as a change in the bursting rate of the spontaneous activity. Jimbo et al. observed similar results with weaker tetani and used voltage clamp to observe inward currents associated with evoked bursts [140]. The intriguing result was that, after tetanization, the onset latencies of these currents resulted were shorter. The following year, Jimbo et al. reported that tetanizing a single electrode resulted in changes in the responses to test pulses delivered from other electrodes [141].

Following this approach, Chiappalone and coworkers designed a more physiological experimental protocol derived from the combination of previous works on associative stimulation for LTD and LTD induction $[153,154]$ and on the results of the Japanese groups $[139,140]$. The applied tetanus was characterized by a sequence of short bursts at $20 \mathrm{~Hz}$ every $5 \mathrm{~s}$ coupled to a stimulation below $1 \mathrm{~Hz}$ for a limited period of time (less than 2 minutes) [20]. The obtained results, based on different pairing of the tetanus with the weak train of stimuli, demonstrated that reliable potentiation was obtained by using more physiological stimulation and without drastically changing the natural spontaneous dynamics of the neuronal system. Moreover the obtained changes were long-lasting since observation up to twenty-four hours after tetanization. 


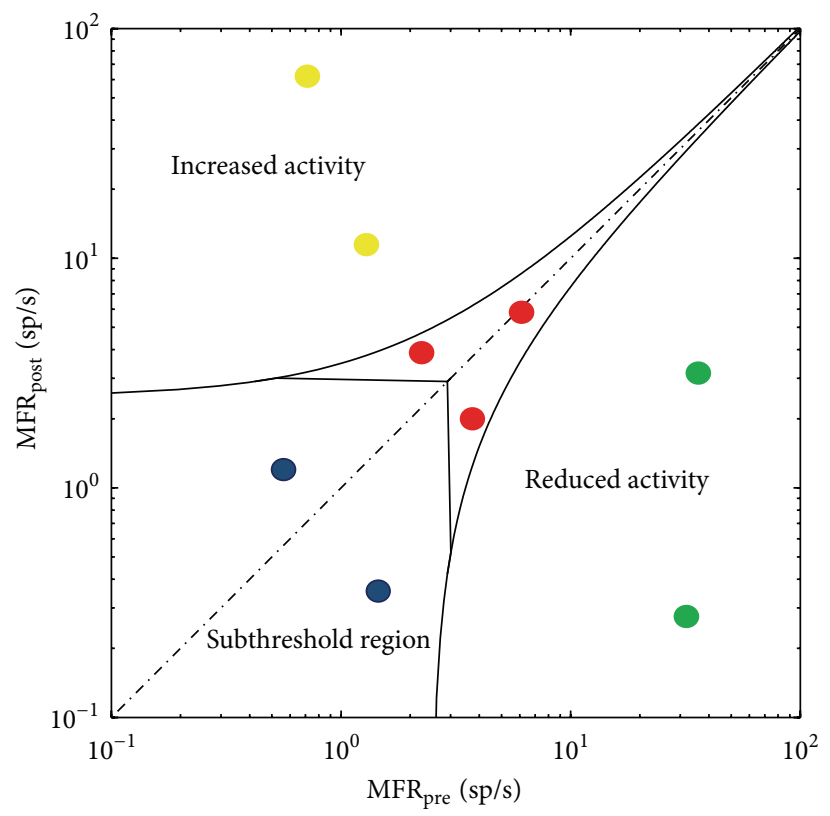

(a)
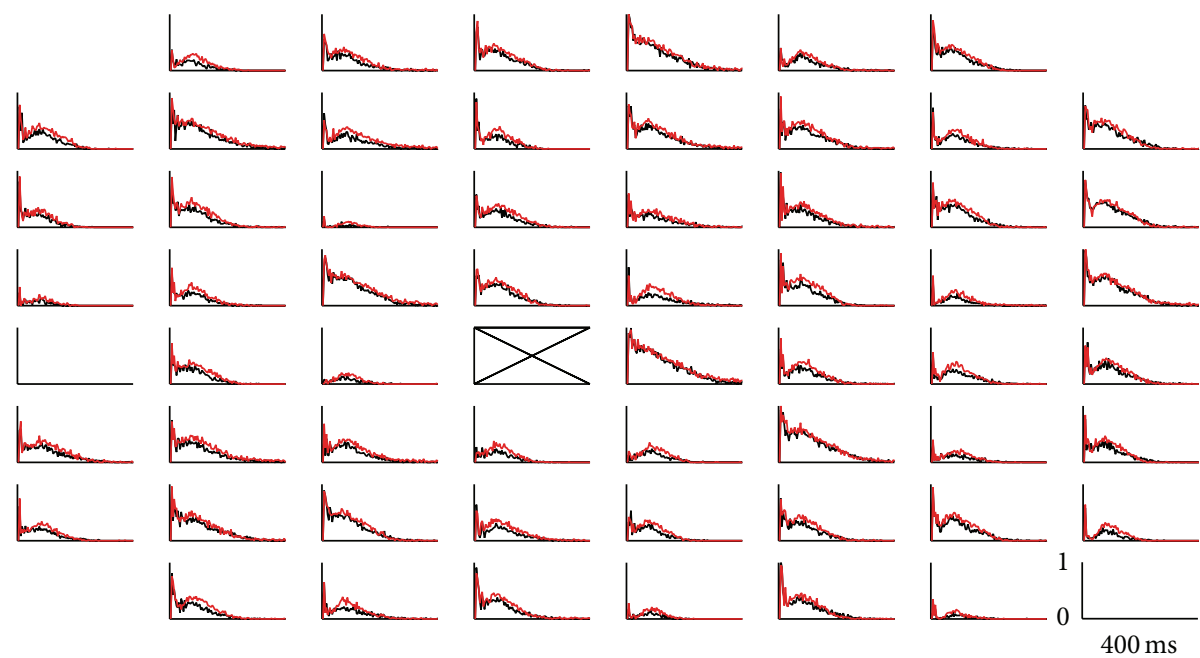

(b)

FIGURE 3: Initial conditions necessary to induce plasticity in cortical cultures. (a) Bilogarithmic plot of the mean spontaneous firing rates of cortical neurons. Spontaneous activity of each network was measured at the beginning of the experiment $\left(\mathrm{MFR}_{\text {pre }}\right)$ and after the first test stimulus session $\left(\mathrm{MFR}_{\text {post }}\right.$ ). Curved lines denote confidence intervals (see [20]). The used colors have the following meanings: red circles represent cultures that verify the MFR stability conditions and are potentially able to show plastic behavior. Blue circles indicate culture with a firing rate too low, while yellow and green circles represent unstable cultures which increase (yellow) or decrease (green) their firing rate. (b) PSTH map. This example shows the effects on the network during the stimulation from the site indicated by the cross. The two superimposed profiles of the PSTH represent the responses of the network to two different sessions of stimulation. Bin size $=4 \mathrm{~ms} ; x$-axis $[0,400] \mathrm{ms} ; y$-axis scale $[0,1]$ is the probability to evoke spikes.

Those findings do not contradict the findings of Jimbo et al. $[140,141]$, according to whom the tetanic stimulation was, by itself, able to induce plasticity in the network. The main result is that the single tetanus is less reliable and it involves a smaller fraction of (effective) connections but its efficacy can be greatly increased when paired with a weak stimulation [20]. A similar work was performed, in 2010, by Le Feber and coworkers [146]. They applied a low-frequency stimulation protocol (i.e., biphasic current pulses at a frequency of 0.2$0.33 \mathrm{~Hz}$ ) to cortical cultures coupled to MEAs. Their analysis investigated possible modifications induced by stimulation on the network functional connectivity. Their main findings showed that stimulation is effective in inducing changes for as long as it triggers network bursts, with very little correlation with the actual stimulation frequency used. Furthermore, stimulation changed network activity patterns from 
the spontaneously observed ones: stimulation-triggered network bursts originate at other points than spontaneously occurring bursts and, therefore, spreading of activity involves different pathways.

The approach followed by the groups led by Torre [144] and Potter $[155,156]$ is completely different and based on more "classical" protocols for inducing plasticity in vitro [14, 157]: in these works, tetanization consists of multisite bursts of stimuli at $250 \mathrm{~Hz}$ or of continuous trains of electrical pulses at $20 \mathrm{~Hz}$ for $15 \mathrm{~min}$. By using such massive, nonphysiological stimulation, the first study [144] reported increase in evoked firing at specific sites, without quantification of duration and amount of the changes. Madhavan et al. [156] reported an increase in global spontaneous activity, without specifically analyzing the evoked response.

\subsection{Limitations and Perspectives on Plasticity in Dissociated} Mammalian Cultures. Inducing plasticity by extracellular electrical stimulation in dissociated mammalian cultures has not been as straightforward as for brain slices [158]. The possible reason behind this could be related to the nonstationary behavior of the dissociated cultures (cf. Section 4.2). Specifically, since much of the activity in cultures is concentrated in bursts (possibly caused by lack of critical neuromodulatory input during development [159]), these dynamics could quickly cancel the effect of plasticity [160]. Moreover synapses are already saturated in culture due to the high density of the established connections.

However, this last motivation could also justify why it has been possible to induce plasticity, as demonstrated by the recent findings reported in this review: assuming a random probability to establish connections and that the chance of forming monosynaptic connections with nearby neurons is quite low [141], there would be a large number of recurrent polysynaptic pathways, as in the intact brain. The firing of a cultured neuron hyperinnervating others can be considered to be analogous to the in vivo situation of synchronous firing of a group of neurons with common targets [149]. For this reason, forms of heterosynaptic plasticity are more likely to occur in dense cultures than homosynaptic ones. For the same reasons, coupled stimulation (e.g., Chiappalone's protocol) mimics, at the cell assembly level, the heterosynaptic pairing as reported for the hippocampus [154], leading to a LTNP, in the form of long-lasting plasticity (i.e., L-LTP) which can be maintained for hours or even days. Figures 4(a) and 4(c) show an example of LTNP. In Figure 4(a) the PSTH shapes of signals from all the electrodes (blue lines) and their average (red thick line) trend are reported before the tetanus delivery (first row) and 1 (middle row) and 24 hours (bottom row) after the tetanus delivery. The tetanic protocol is sketched in panel (b). Qualitatively, a change in the shape of the response can be appreciated. To quantify such results, Figure 4(c) plots the area under the PSTH curves before tetanus delivery (black squares) and 1 (red squares) and 24 hours (green squares) after the tetanus delivery. A clear potentiation of the network can be observed and quantified by the slopes of the linear fittings (dashed lines). As reported in [149], synaptic plasticity at the network level provides a distributed mechanism to convert and store temporal information into spatially distributed patterns of synaptic modification.

\section{Plasticity in Closed-Loop Experiments}

Closed-loop experiments on in vitro neural networks have been introduced to investigate whether such preparations could perform a learning task without the need for a separate rewarding entity: in 2001, the group of Marom [143] implemented a relatively simple activity-dependent stimulation protocol that would cease once the firing of the network met a predefined threshold: they developed an activitydependent adaptive stimulation protocol, aimed at training a culture to produce a predefined response upon stimulation. In this way, networks could be taught to respond in specific ways to test pulses, by repeatedly stimulating them until the desired response was obtained. This approach was based on general learning theories (stimulus regulation principle). In their experiments, the reward consisted in a reduction of the driving stimulus, precluding the acquisition of any new stimulus-response associations.

Similar experiments have been more recently conducted by the groups of Le Feber [146] and Kazantsev [161], in 2010 and 2013, respectively. In more detail, an electrode in a standard, 60-electrode MEA layout was used to deliver lowfrequency $(\leq 1 \mathrm{~Hz})$ stimulation pulses. At the same time, the strength of functional connectivity between any pair of electrodes is estimated in real time, with stimulation protocols being interrupted when the functional connectivity between the stimulating electrode and a different arbitrarily chosen electrode in the MEA showed a significant increase. The results of these experimental campaigns proved that indeed closed-loop stimulation may be used to induce specific changes in network connectivity with no or very little a priori knowledge of the network structure.

Along the same line, Potter's group induced random changes in synaptic connections by stimulating in quick succession from several different electrodes, in order to induce a mix of potentiation and depression [162]. A stabilization pattern also consisted in stimulation from different electrodes, but interpulse intervals were chosen in order not to trigger spike timing dependent plasticity (STDP). In those experiments, the activity of the network resulted in the movement of an "animat" (i.e., an artificial animal): each time the behavior of the animat approached the intended one, the stabilization pattern was delivered; conversely, the network was stimulated with the training pattern to provoke a change of the animat behavior. The main result of that work was that the same patterns used for training did not cause any significant plasticity if delivered in open loop. However, this was not the only example of hybrid neural-artificial systems controlling a robot in closed loop. Several neurorobotic systems have been introduced and presented in the literature between 2007 and 2012 [163-166]. Differently from Potter's, those systems did rely on a priori knowledge of connectivity within the neural networks to successfully drive the robot past obstacles. These systems implement a closed-loop system that takes inspiration from a typical physiological sensorymotor loop: the sensors of the robot gather information about 

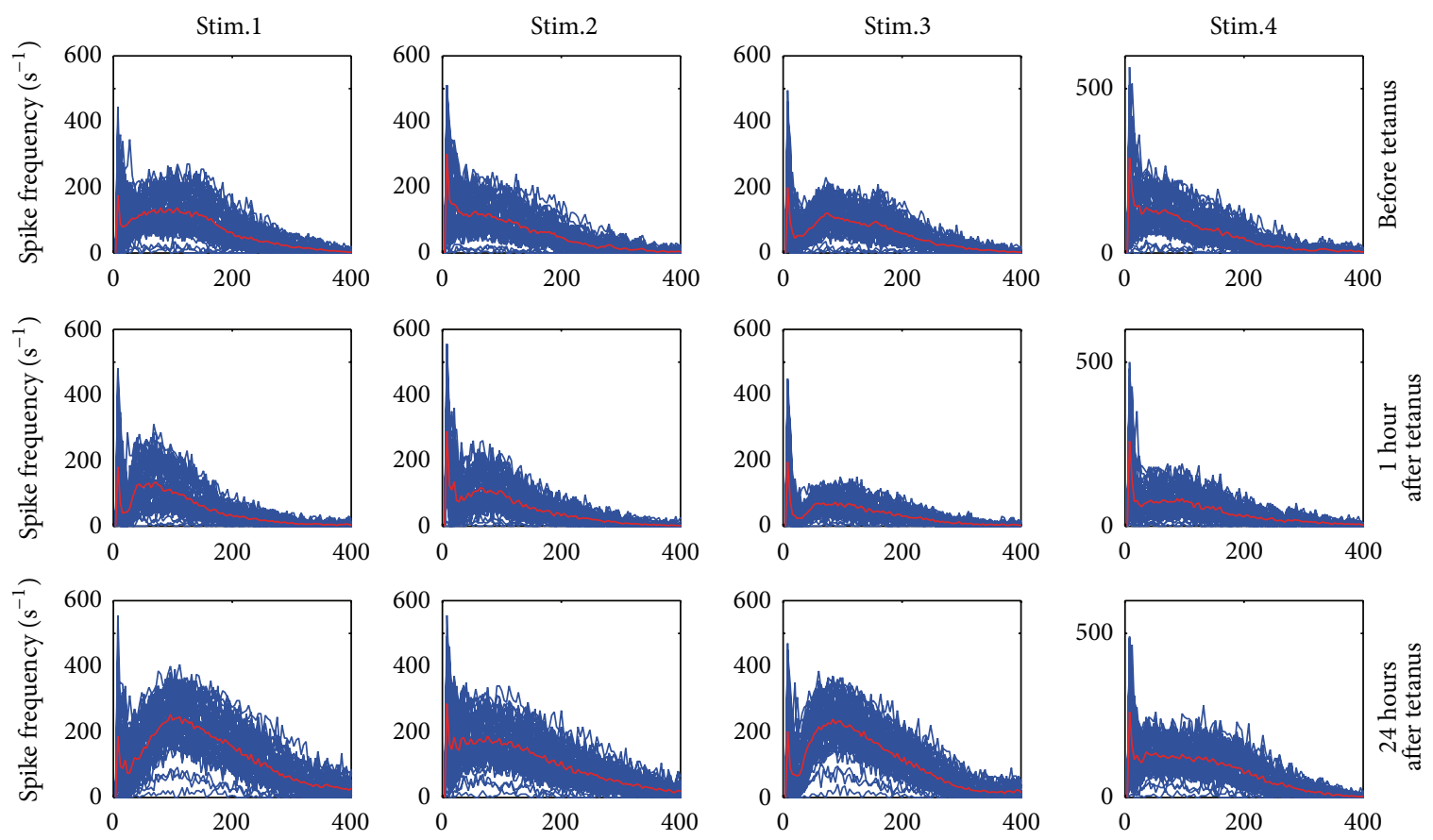

Time relative to stimulus (ms) Time relative to stimulus (ms)

(a)

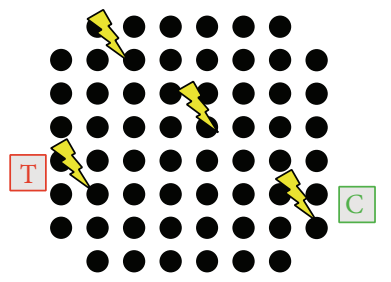

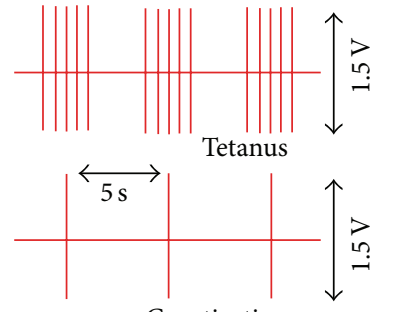

Coactivation

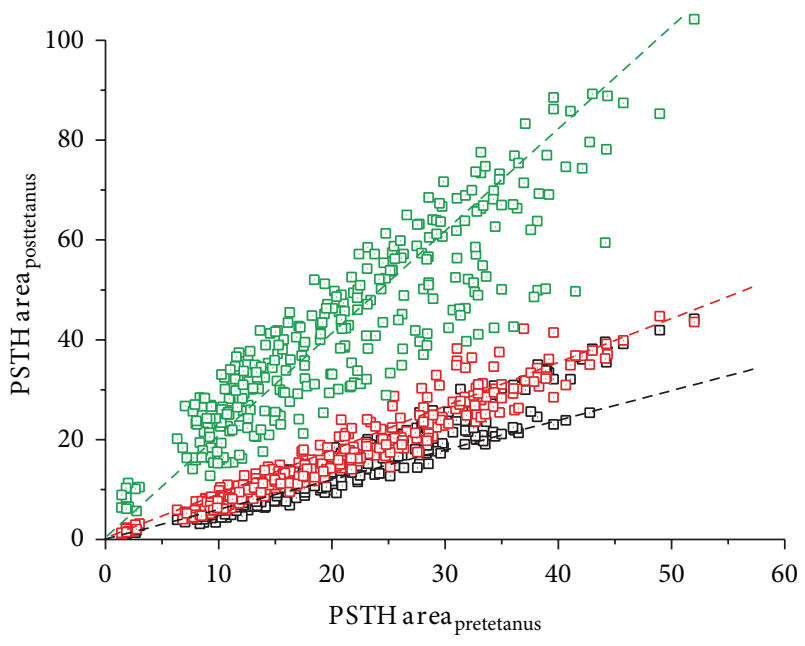

$\square$ Before tetanus

$\square 24$ hours after tetanus

$\square 1$ hour after tetanus

(b)

(c)

FIgURE 4: (a) Superimposition of the PSTH curves (each per electrode). The thick red line indicates the average shape. Each row indicates a peculiar phase of the protocol: before the delivery of the tetanus (first row) and 1 hour (second row) and 24 hours (third row) after delivery of the tetanus. Each column indicates a different stimulation site. (b) Sketch of the tetanic protocol described in [20]. Each black dot represents a microelectrode. The yellow arrows indicate where the stimuli are delivered. The tetanic protocol consists of a high frequency tetanic stimulation coupled to a low-frequency (in-phase) stimulation. (c) Scatter plot of the PSTH area for each active electrode. Each point represents an active channel with a certain PSTH area during three phases of the protocol: before the delivery of the tetanus (black squares) and 1 hour (red squares) and 24 hours (green squares) after delivery of the tetanus. The dashed lines represent the linear fitting of the three scatter plots. 
the surrounding obstacles, which is coded as a sequence of electrical stimuli delivered to the neural networks. The responses of the network are in turn decoded and used to safely drive the robot around its environment. These systems are generally intended as platforms for the study of specific aspects of neural network mechanisms, such as coding and decoding, or to test novel learning paradigms.

Identification of input-output relationships even for single neurons has proven intractable for a long time, given the timescales and nonlinearities involved. A step forward in this direction has been taken in 2011, when Marom's group introduced for the first time the concept of "neuronal response clamp" [167]. The idea is that of taking advantage of a proportional-integral-derivative (PID) controller to modulate the amplitude of a fixed rate electrical stimulation in order to maintain a stable response ratio at a given neuron. In this way, it is possible to estimate the threshold of the neuron, a high-level, functionally relevant variable. Thanks to the closed-loop neuronal clamp, in 2012 the same group investigated the interplay between network burst and singleneuron threshold [168]. They found the two phenomena to be deeply intertwined: the size of bursts results correlated with threshold values at the time of inset, while the dynamics of threshold recovery govern both spontaneous bursting and response to electrical stimulation. The effect of closed-loop stimulation on plasticity is presented in a more recent work by the same group [169]. They concluded that, in open-loop conditions, networks will respond to electrical stimulation whenever they have built up enough resources to do so; on the other hand, in a closed loop, networks are forced to keep up with stimulation rate: this imposes the recruitment of resources which are generally left unused. In turn, this leads to enhanced changes in connectivity compared to those observed in open-loop experiments.

\section{Concluding Remarks}

Simultaneous multisite long-lasting recordings with MEAs have opened new perspectives in the studies of formation and dynamics of complex neural networks allowing detailed investigations at the level of single cells and at population scales both in vitro and in vivo. In the last decades, technical improvements have increased spatial resolution of MEA recordings [36] and recently the detection of subthreshold signals such as synaptic potentials has been demonstrated in invertebrate neurons [97]. Future efforts will be focused on the development of strategies to record synaptic signals in mammalian neurons too, as well as to create more realistic connectivity, like 3D structures [170] or the presence of heterogeneous neuronal populations [171]. Studies of connectivity and synaptic plasticity in dissociated mammalian cultures will help to understand complex dynamics underlying both physiological behaviors and pathological alterations [172] associated with changes in firing patterns of large populations of neurons as it happens in epileptic disorders.

As reviewed in this work, the behavior of dissociated cortical networks can be shaped by the delivery of ad hoc stimulation patterns suggesting that this reduced in vitro experimental model is capable of learning or adapting to the timing of the stimuli. These conclusions have been extended in 2010 by Buonamano and coworkers that found that neuronal dynamics in a complex circuit can be modified through experience and that the temporal structure of such expressed dynamics reflects the temporal interval used during training [173].

Finally, the possibility to interface neuronal networks with MEAs allows the realization of neurorobotic frameworks. Such hybrid platforms are a valid tool for the study of mechanisms of neural coding and the computational and adaptive properties of neuronal assemblies [174]. In a longterm vision, these systems could be used to better understand neural pathologies, to design neural prosthetics, and to create different types of hybrid intelligence.

\section{Conflict of Interests}

The authors declare that there is no conflict of interests regarding the publication of this paper.

\section{References}

[1] R. W. Sperry, "Optic nerve regeneration with return of vision in anurans," Journal of Neurophysiology, vol. 7, no. 1, pp. 57-69, 1944.

[2] R. Sperry, "Restoration of vision after crossing of optic nerves and after contralateral transplantation of eye," Journal of Neurophysiology, vol. 8, no. 1, pp. 15-28, 1945.

[3] B. Grafstein, "Roger Sperry: pioneer of neuronal specificity", Journal of Neurophysiology, vol. 96, no. 6, pp. 2827-2829, 2006.

[4] R. Christensen, Z. Shao, and D. A. Colón-Ramos, "The cell biology of synaptic specificity during development," Current Opinion in Neurobiology, vol. 23, no. 6, pp. 1018-1026, 2013.

[5] M. E. Williams, J. de Wit, and A. Ghosh, "Molecular mechanisms of synaptic specificity in developing neural circuits," Neuron, vol. 68, no. 1, pp. 9-18, 2010.

[6] S. Yogev and K. Shen, "Cellular and molecular mechanisms of synaptic specificity," Annual Review of Cell and Developmental Biology, vol. 30, no. 1, pp. 417-437, 2014.

[7] D. H. Hubel and T. N. Wiesel, "Binocular interaction in striate cortex of kittens reared with artificial squint," Journal of Neurophysiology, vol. 28, no. 6, pp. 1041-1059, 1965.

[8] T. N. Wiesel and D. H. Hubel, "Effects of visual deprivation on morphology and physiology of cells in the cats lateral geniculate body," Journal of Neurophysiology, vol. 26, pp. 978-993, 1963.

[9] L. C. Katz and C. J. Shatz, "Synaptic activity and the construction of cortical circuits," Science, vol. 274, no. 5290, pp. 1133-1138, 1996.

[10] J. S. Espinosa and M. P. Stryker, "Development and plasticity of the primary visual cortex," Neuron, vol. 75, no. 2, pp. 230-249, 2012.

[11] N. Yamamoto and G. López-Bendito, "Shaping brain connections through spontaneous neural activity," European Journal of Neuroscience, vol. 35, no. 10, pp. 1595-1604, 2012.

[12] K. Ganguly and M.-M. Poo, "Activity-dependent neural plasticity from bench to bedside," Neuron, vol. 80, no. 3, pp. 729-741, 2013.

[13] D. O. Hebb, Organization of Behavior, John Wiley \& Sons, New York, NY, USA, 1949. 
[14] T. V. P. Bliss and T. Lomo, "Long lasting potentiation of synaptic transmission in the dentate area of the anaesthetized rabbit following stimulation of the perforant path," Journal of Physiology, vol. 232, no. 2, pp. 331-356, 1973.

[15] M. Ito and M. Kano, "Long-lasting depression of parallel fiberPurkinje cell transmission induced by conjunctive stimulation of parallel fibers and climbing fibers in the cerebellar cortex," Neuroscience Letters, vol. 33, no. 3, pp. 253-258, 1982.

[16] M. Mayford, S. A. Siegelbaum, and E. R. Kandel, "Synapses and memory storage," Cold Spring Harbor Perspectives in Biology, vol. 4, no. 6, Article ID a005751, 2012.

[17] E. R. Kandel, Y. Dudai, and M. R. Mayford, "The molecular and systems biology of memory," Cell, vol. 157, no. 1, pp. 163-186, 2014.

[18] P. Caroni, F. Donato, and D. Muller, "Structural plasticity upon learning: regulation and functions," Nature Reviews Neuroscience, vol. 13, no. 7, pp. 478-490, 2012.

[19] P. Massobrio, C. N. G. Giachello, M. Ghirardi, and S. Martinoia, "Selective modulation of chemical and electrical synapses of Helix neuronal networks during in vitro development," $B M C$ Neuroscience, vol. 14, article 22, 2013.

[20] M. Chiappalone, P. Massobrio, and S. Martinoia, "Network plasticity in cortical assemblies," European Journal of Neuroscience, vol. 28, no. 1, pp. 221-237, 2008.

[21] A. Mazzoni, F. D. Broccard, E. Garcia-Perez, P. Bonifazi, M. E. Ruaro, and V. Torre, "On the dynamics of the spontaneous activity in neuronal networks," PLoS ONE, vol. 2, no. 5, article e439, 2007.

[22] P. Massobrio, M. Tedesco, C. Giachello, M. Ghirardi, F. Fiumara, and S. Martinoia, "Helix neuronal ensembles with controlled cell type composition and placement develop functional polysynaptic circuits on Micro-Electrode Arrays," Neuroscience Letters, vol. 467, no. 2, pp. 121-126, 2009.

[23] V. Pasquale, P. Massobrio, L. L. Bologna, M. Chiappalone, and S. Martinoia, "Self-organization and neuronal avalanches in networks of dissociated cortical neurons," Neuroscience, vol. 153, no. 4, pp. 1354-1369, 2008.

[24] G. J. Brewer, M. D. Boehler, A. N. Ide, and B. C. Wheeler, "Chronic electrical stimulation of cultured hippocampal networks increases spontaneous spike rates," Journal of Neuroscience Methods, vol. 184, no. 1, pp. 104-109, 2009.

[25] D. A. Wagenaar, J. Pine, and S. M. Potter, "An extremely rich repertoire of bursting patterns during the development of cortical cultures," BMC Neuroscience, vol. 7, article 11, 2006.

[26] P. Massobrio, P. L. Baljon, A. Maccione, M. Chiappalone, and S. Martinoia, "Activity modulation elicited by electrical stimulation in networks of dissociated cortical neurons," in Proceedings of the IEEE Conference on Engineering in Medicine and Biology Society (EMBC '07), pp. 3008-3011, Lyon, France, August 2007.

[27] D. A. Wagenaar, R. Madhavan, J. Pine, and S. M. Potter, "Controlling bursting in cortical cultures with closed-loop multielectrode stimulation," The Journal of Neuroscience, vol. 25, no. 3, pp. 680-688, 2005.

[28] D. A. Wagenaar, J. Pine, and S. M. Potter, "Effective parameters for stimulation of dissociated cultures using multi-electrode arrays," Journal of Neuroscience Methods, vol. 138, no. 1-2, pp. 27-37, 2004.

[29] G. W. Gross, B. Rhoades, and R. Jordan, "Neuronal networks for biochemical sensing," Sensors and Actuators: B. Chemical, vol. 6, no. 1-3, pp. 1-8, 1992.
[30] G. W. Gross, B. K. Rhoades, H. M. E. Azzazy, and M.-C. Wu, "The use of neuronal networks on multielectrode arrays as biosensors," Biosensors and Bioelectronics, vol. 10, no. 6-7, pp. 553$567,1995$.

[31] S. Martinoia, L. Bonzano, M. Chiappalone, and M. Tedesco, "Electrophysiological activity modulation by chemical stimulation in networks of cortical neurons coupled to microelectrode arrays: a biosensor for neuropharmacological applications," Sensors and Actuators, B: Chemical, vol. 108, no. 1-2, pp. 589596, 2005.

[32] U. Frey, U. Egert, F. Heer, S. Hafizovic, and A. Hierlemann, "Microelectronic system for high-resolution mapping of extracellular electric fields applied to brain slices," Biosensors and Bioelectronics, vol. 24, no. 7, pp. 2191-2198, 2009.

[33] S. Hafizovic, F. Heer, T. Ugniwenko et al., "A CMOS-based microelectrode array for interaction with neuronal cultures," Journal of Neuroscience Methods, vol. 164, no. 1, pp. 93-106, 2007.

[34] K. Imfeld, S. Neukom, A. Maccione et al., "Large-scale, highresolution data acquisition system for extracellular recording of electrophysiological activity," IEEE Transactions on Biomedical Engineering, vol. 55, no. 8, pp. 2064-2073, 2008.

[35] B. Eversmann, M. Jenkner, F. Hofmann et al., "A 128 x 128 CMOS biosensor array for extracellular recording of neural activity," IEEE Journal of Solid-State Circuits, vol. 38, no. 12, pp. 23062317, 2003.

[36] L. Berdondini, K. Imfeld, A. MacCione et al., "Active pixel sensor array for high spatio-temporal resolution electrophysiological recordings from single cell to large scale neuronal networks," Lab on a Chip-Miniaturisation for Chemistry and Biology, vol. 9, no. 18, pp. 2644-2651, 2009.

[37] P. Fromherz, A. Offenhäusser, T. Vetter, and J. Weis, "A neuronsilicon junction: a Retzius cell of the leech on an insulated-gate field-effect transistor," Science, vol. 252, no. 5010, pp. 1290-1293, 1991.

[38] S. Marom and G. Shahaf, "Development, learning and memory in large random networks of cortical neurons: lessons beyond anatomy," Quarterly Reviews of Biophysics, vol. 35, no. 1, pp. 6387, 2002.

[39] M. Taketani and M. Baudry, Eds., Advances in Network Electrophysiology: Using Multi-Electrode Array, Springer, New York, NY, USA, 2006.

[40] R. Schätzthauer, "Neuron-silicon junction with voltage-gated ionic currents," European Journal of Neuroscience, vol. 10, no. 6, pp. 1956-1962, 1998.

[41] S. Vassanelli and P. Fromherz, "Transistor-records of excitable neurons from rat brain," Applied Physics A: Materials Science and Processing, vol. 66, no. 4, pp. 459-463, 1998.

[42] A. Elshabini-Riad and F. D. Barlow, Thin Film Technology Handbook, McGraw-Hill, New York, NY, USA, 1998.

[43] S. Vassaneili and P. Fromherz, "Transistor probes local potassium conductances in the adhesion region of cultured rat hippocampal neurons," The Journal of Neuroscience, vol. 19, no. 16, pp. 6767-6773, 1999.

[44] M. Jenkner, B. Müller, and P. Fromherz, "Interfacing a silicon chip to pairs of snail neurons connected by electrical synapses," Biological Cybernetics, vol. 84, no. 4, pp. 239-249, 2001.

[45] G. Zeck and P. Fromherz, "Noninvasive neuroelectronic interfacing with synaptically connected snail neurons immobilized on a semiconductor chip," Proceedings of the National Academy of Sciences of the United States of America, vol. 98, no. 18, pp. 10457-10462, 2001. 
[46] P. Fromherz, "Neuroelectronic interfacing: semiconductor chips with ion channels, nerve cells, and brain," in Nanoelectronics and Information Technology, R. Waser, Ed., pp. 781-810, Wiley-VCH, Berlin, Germany, 2003.

[47] M. Voelker and P. Fromherz, "Signal transmission from individual mammalian nerve cell to field-effect transistor," Small, vol. 1, no. 2, pp. 206-210, 2005.

[48] T. Gabay, E. Jakobs, E. Ben-Jacob, and Y. Hanein, "Engineered self-organization of neural networks using carbon nanotube clusters," Physica A, vol. 350, no. 2-4, pp. 611-621, 2005.

[49] T. Gabay, M. Ben-David, I. Kalifa et al., "Electro-chemical and biological properties of carbon nanotube based multi-electrode arrays," Nanotechnology, vol. 18, no. 3, Article ID 035201, 2007.

[50] V. Lovat, D. Pantarotto, L. Lagostena et al., "Carbon nanotube substrates boost neuronal electrical signaling," Nano Letters, vol. 5, no. 6, pp. 1107-1110, 2005.

[51] A. Mazzatenta, M. Giugliano, S. Campidelli et al., "Interfacing neurons with carbon nanotubes: electrical signal transfer and synaptic stimulation in cultured brain circuits," The Journal of Neuroscience, vol. 27, no. 26, pp. 6931-6936, 2007.

[52] G. Massobrio, P. Massobrio, and S. Martinoia, "Modeling the neuron-carbon Nanotube-ISFET junction to investigate the electrophysiological neuronal activity," Nano Letters, vol. 8, no. 12, pp. 4433-4440, 2008.

[53] E. W. Keefer, B. R. Botterman, M. I. Romero, A. F. Rossi, and G. W. Gross, "Carbon nanotube coating improves neuronal recordings," Nature Nanotechnology, vol. 3, no. 7, pp. 434-439, 2008.

[54] K. Wang, H. A. Fishman, H. Dai, and J. S. Harris, "Neural stimulation with a carbon nanotube microelectrode array," Nano Letters, vol. 6, no. 9, pp. 2043-2048, 2006.

[55] A. Hai, J. Shappir, and M. E. Spira, "In-cell recordings by extracellular microelectrodes," Nature Methods, vol. 7, no. 3, pp. 200202, 2010.

[56] A. Hai, A. Dormann, J. Shappir et al., "Spine-shaped gold protrusions improve the adherence and electrical coupling of neurons with the surface of micro-electronic devices," Journal of the Royal Society Interface, vol. 6, no. 41, pp. 1153-1165, 2009.

[57] N. Schmold and N. I. Syed, "Molluscan neurons in culture: shedding light on synapse formation and plasticity," Journal of Molecular Histology, vol. 43, no. 4, pp. 383-399, 2012.

[58] I. D. Dietzel, P. Drapeau, and J. G. Nicholls, "Voltage dependence of 5-hydroxytryptamine release at a synapse between identified leech neurones in culture," Journal of Physiology, vol. 372, pp. 191-205, 1986.

[59] P. Bonifazi and P. Fromherz, "Silicon chip for electronic communication between nerve cells by noninvasive interfacing and analog-digital processing," Advanced Materials, vol. 14, pp. 1190-1193, 2002.

[60] R. L. Ridgway, N. I. Syed, K. Lukowiak, and A. G. M. Bulloch, "Nerve growth factor (NGF) induces sprouting of specific neurons of the snail, Lymnaea stagnalis," Journal of Neurobiology, vol. 22, no. 4, pp. 377-390, 1991.

[61] A. A. Prinz and P. Fromherz, "Electrical synapses by guided growth of cultured neurons from the snail Lymnaea stagnalis," Biological Cybernetics, vol. 82, no. 4, pp. L1-L5, 2000.

[62] R. A. Kaul, N. I. Syed, and P. Fromherz, "Neuron-semiconductor chip with chemical synapse between identified neurons," Physical Review Letters, vol. 92, no. 3, Article ID 038102, 2004.
[63] T. Hamakawa, M. A. Woodin, M. C. Bjorgum et al., "Excitatory synaptogenesis between identified Lymnaea neurons requires extrinsic trophic factors and is mediated by receptor tyrosine kinases," The Journal of Neuroscience, vol. 19, no. 21, pp. 93069312, 1999.

[64] A. B. Smit, N. I. Syed, D. Schaap et al., "A glia-derived acetylcholine-binding protein that modulates synaptic transmission," Nature, vol. 411, no. 6835, pp. 261-268, 2001.

[65] M. A. Woodin, T. Hamakawa, M. Takasaki, K. Lukowiak, and N. I. Syed, "Trophic factor-induced plasticity of synaptic connections between identified Lymnaea neurons," Learning and Memory, vol. 6, no. 3, pp. 307-316, 1999.

[66] Z.-P. Feng, J. Klumperman, K. Lukowiak, and N. I. Syed, "In vitro synaptogenesis between the somata of identified Lymnaea neurons requires protein synthesis but not extrinsic growth factors or substrate adhesion molecules," The Journal of Neuroscience, vol. 17, no. 20, pp. 7839-7849, 1997.

[67] A. Hasenstaub, Y. Shu, B. Haider, U. Kraushaar, A. Duque, and D. A. McCormick, "Inhibitory postsynaptic potentials carry synchronized frequency information in active cortical networks," Neuron, vol. 47, no. 3, pp. 423-435, 2005.

[68] D. W. Munno and N. I. Syed, "Synaptogenesis in the CNS: an odyssey from wiring together to firing together," Journal of Physiology, vol. 552, part 1, pp. 1-11, 2003.

[69] H. Naruo, S. Onizuka, D. Prince, M. Takasaki, and N. I. Syed, "Sevoflurane blocks cholinergic synaptic transmission postsynaptically but does not affect short-term potentiation," Anesthesiology, vol. 102, no. 5, pp. 920-928, 2005.

[70] C. C. Luk, H. Naruo, D. Prince et al., "A novel form of presynaptic CaMKII-dependent short-term potentiation between Lymnaea neurons," European Journal of Neuroscience, vol. 34, no. 4, pp. 569-577, 2011.

[71] D. M. Wilson, "The central nervous control of flight in a locust," The Journal of Experimental Biology, vol. 38, pp. 471-490, 1961.

[72] F. Delcomyn, "Neural basis of rhythmic behavior in animals," Science, vol. 210, no. 4469, pp. 492-498, 1980.

[73] E. Marder and R. L. Calabrese, "Principles of rhythmic motor pattern generation," Physiological Reviews, vol. 76, no. 3, pp. 687-717, 1996.

[74] C. J. H. Elliott and A. J. Susswein, "Comparative neuroethology of feeding control in molluscs," Journal of Experimental Biology, vol. 205, no. 7, pp. 877-896, 2002.

[75] C. A. Harris, P. A. Passaro, I. Kemenes, G. Kemenes, and M. O'Shea, "Sensory driven multi-neuronal activity and associative learning monitored in an intact CNS on a multielectrode array," Journal of Neuroscience Methods, vol. 186, no. 2, pp. 171-178, 2010.

[76] T. E. Audesirk, J. E. Alexander Jr., G. J. Audesirk, and C. M. Moyer, "Rapid, nonaversive conditioning in a freshwater gastropod. I. Effects of age and motivation," Behavioral and Neural Biology, vol. 36, no. 4, pp. 379-390, 1982.

[77] S. Kojima, M. Yamanaka, Y. Fujito, and E. Ito, "Differential neuroethological effects of aversive and appetitive reinforcing stimuli on associative learning in Lymnaea stagnalis," Zoological Science, vol. 13, no. 6, pp. 803-812, 1996.

[78] K. Lukowiak, E. Ringseis, G. Spencer, W. Wildering, and N. Syed, "Operant conditioning of aerial respiratory behaviour in Lymnaea stagnalis," Journal of Experimental Biology, vol. 199, no. 3, pp. 683-691, 1996. 
[79] K. Lukowiak, R. Cotter, J. Westly, E. Ringseis, G. Spencer, and N. Syed, "Long-term memory of an operantly conditioned respiratory behaviour pattern in Lymnaea stagnalis," Journal of Experimental Biology, vol. 201, no. 6, pp. 877-882, 1998.

[80] K. Staras, G. Kemenes, and P. R. Benjamin, "Neurophysiological correlates of unconditioned and conditioned feeding behavior in the pond snail Lymnaea stagnalis," Journal of Neurophysiology, vol. 79, no. 6, pp. 3030-3040, 1998.

[81] K. Staras, G. Kemenes, and P. R. Benjamin, "Cellular traces of behavioral classical conditioning can be recorded at several specific sites in a simple nervous system," The Journal of Neuroscience, vol. 19, no. 1, pp. 347-357, 1999.

[82] G. E. Spencer, M. H. Kazmi, N. I. Syed, and K. Lukowiak, "Changes in the activity of a CPG neuron after the reinforcement of an operantly conditioned behavior in Lymnaea," Journal of Neurophysiology, vol. 88, no. 4, pp. 1915-1923, 2002.

[83] G. Kemenes, K. Staras, and P. R. Benjamin, "In vitro appetitive classical conditioning of the feeding response in the pond snail Lymnaea stagnalis," Journal of Neurophysiology, vol. 78, no. 5, pp. 2351-2362, 1997.

[84] M. Marra, I. Kemenes, J. S. Ireland, M. O'Shea, and P. R. Benjamin, Time-Course of Electrical and Molecular Changes Following One-Trial in Vitro Chemical Conditioning, Society for Neuroscience, San Diego, Calif, USA, 2007.

[85] C. A. Harris, C. L. Buckley, T. Nowotny et al., "Multi-neuronal refractory period adapts centrally generated behaviour to reward," PLoS ONE, vol. 7, no. 7, Article ID e42493, 2012.

[86] P. Darbon, L. Scicluna, A. Tscherter, and J. Streit, "Mechanisms controlling bursting activity induced by disinhibition in spinal cord networks," European Journal of Neuroscience, vol. 15, no. 4, pp. 671-683, 2002.

[87] P. Darbon, A. Tscherter, C. Yvon, and J. Streit, "Role of the electrogenic $\mathrm{Na} / \mathrm{K}$ pump in disinhibition-induced bursting in cultured spinal networks," Journal of Neurophysiology, vol. 90, no. 5, pp. 3119-3129, 2003.

[88] B. Fedirchuk, P. Wenner, P. J. Whelan, S. Ho, J. Tabak, and M. J. O’Donovan, "Spontaneous network activity transiently depresses synaptic transmission in the embryonic chick spinal cord," The Journal of Neuroscience, vol. 19, no. 6, pp. 2102-2112, 1999.

[89] R. A. Wise, "Role of brain dopamine in food reward and reinforcement," Philosophical Transactions of the Royal Society of London, Series B: Biological Sciences, vol. 361, no. 1471, pp. 11491158, 2006.

[90] M. D. Johnson, R. K. Franklin, K. A. Scott, R. B. Brown, and D. R. Kipke, "Neural probes for concurrent detection of neurochemical and electrophysiological signals in vivo," in Proceedings of the 27th Annual International Conference of the Engineering in Medicine and Biology Society, vol. 7, pp. 7325-7328, IEEE, Shanghai, China, January 2006.

[91] M. D. Johnson, R. K. Franklin, M. D. Gibson, R. B. Brown, and D. R. Kipke, "Implantable microelectrode arrays for simultaneous electrophysiological and neurochemical recordings," Journal of Neuroscience Methods, vol. 174, no. 1, pp. 62-70, 2008.

[92] Y. Song, N. Lin, C. Liu, H. Jiang, G. Xing, and X. Cai, "A novel dual mode microelectrode array for neuroelectrical and neurochemical recording in vitro," Biosensors \& Bioelectronics, vol. 38, no. 1, pp. 416-420, 2012.

[93] M.-C. Chuang, H.-Y. Lai, J.-A. Annie Ho, and Y.-Y. Chen, "Multifunctional microelectrode array (mMEA) chip for neuralelectrical and neural-chemical interfaces: characterization of comb interdigitated electrode towards dopamine detection," Biosensors and Bioelectronics, vol. 41, no. 1, pp. 602-607, 2013.
[94] J. G. Hensler, F. Artigas, A. Bortolozzi et al., "Catecholamine/ serotonin interactions: systems thinking for brain function and disease," Advances in Pharmacology, vol. 68, pp. 167-197, 2013.

[95] M. A. Kurian, P. Gissen, M. Smith, S. J. Heales, and P. T. Clayton, "The monoamine neurotransmitter disorders: an expanding range of neurological syndromes," The Lancet Neurology, vol. 10, no. 8, pp. 721-733, 2011.

[96] B. A. Patel, C. C. Luk, P. L. Leow, A. J. Lee, W. Zaidi, and N. I. Syed, "A planar microelectrode array for simultaneous detection of electrically evoked dopamine release from distinct locations of a single isolated neuron," Analyst, vol. 138, no. 10, pp. 2833 2839, 2013.

[97] M. E. Spira and A. Hai, "Multi-electrode array technologies for neuroscience and cardiology," Nature Nanotechnology, vol. 8, no. 2, pp. 83-94, 2013.

[98] M. E. Spira, D. Kamber, A. Dormann et al., "Improved neuronal adhesion to the surface of electronic device by engulfment of protruding micro-nails fabricated on the chip surface," in Proceedings of the 4th International Conference on SolidState Sensors, Actuators and Microsystems (Transducers and Eurosensors '07), pp. 1247-1250, June 2007.

[99] A. Hai, D. Kamber, G. Malkinson et al., "Changing gears from chemical adhesion of cells to flat substrata toward engulfment of micro-protrusions by active mechanisms," Journal of neural engineering, vol. 6, no. 6, p. 66009, 2009.

[100] A. Hai, J. Shappir, and M. E. Spira, "Long-term, multisite, parallel, in-cell recording and stimulation by an array of extracellular microelectrodes," Journal of Neurophysiology, vol. 104, no. 1, pp. 559-568, 2010.

[101] S. Schacher and E. Proshansky, "Neurite regeneration by Aplysia neurons in dissociated cell culture: Modulation by Aplysia hemolymph and the presence of the initial axonal segment," The Journal of Neuroscience, vol. 3, no. 12, pp. 2403-2413, 1983.

[102] M. E. Spira, A. Dormann, U. Ashery et al., "Use of Aplysin neurons for the study of cellular alterations and the resealing of transected axons in vitro," Journal of Neuroscience Methods, vol. 69, no. 1, pp. 91-102, 1996.

[103] C. H. Bailey and E. R. Kandel, "Chapter 10 Synaptic remodeling, synaptic growth and the storage of long-term memory in Aplysia," Progress in Brain Research, vol. 169, pp. 179-198, 2008.

[104] M. Ghirardi, A. Casadio, L. Santarelli, and P. G. Montarolo, "Aplysia hemolymph promotes neurite outgrowth and synaptogenesis of identified Helix neurons in cell culture," Invertebrate Neuroscience, vol. 2, no. 1, pp. 41-49, 1996.

[105] F. Fiumara, G. Leitinger, C. Milanese, P. G. Montarolo, and M. Ghirardi, "In vitro formation and activity-dependent plasticity of synapses between Helix neurons involved in the neural control of feeding and withdrawal behaviors," Neuroscience, vol. 134, no. 4, pp. 1133-1151, 2005.

[106] C. N. G. Giachello, F. Fiumara, C. Giacomini et al., "MAPK/Erkdependent phosphorylation of synapsin mediates formation of functional synapses and short-term homosynaptic plasticity," Journal of Cell Science, vol. 123, no. 6, pp. 881-893, 2010.

[107] C. N. G. Giachello, P. G. Montarolo, and M. Ghirardi, "Synaptic functions of invertebrate varicosities: what molecular mechanisms lie beneath," Neural Plasticity, vol. 2012, Article ID 670821, 14 pages, 2012.

[108] E. Claverol-Tinture, J. Cabestany, and X. Rosell, "Multisite recording of extracellular potentials produced by microchannel-confined neurons in-vitro," IEEE Transactions on Biomedical Engineering, vol. 54, no. 2, pp. 331-335, 2007. 
[109] E. Claverol-Tinturé, M. Ghirardi, F. Fiumara, X. Rosell, and J. Cabestany, "Multielectrode arrays with elastomeric microstructured overlays for extracellular recordings from patterned neurons," Journal of Neural Engineering, vol. 2, no. 2, pp. L1-L7, 2005.

[110] M. Garofalo, T. Nieus, P. Massobrio, and S. Martinoia, "Evaluation of the performance of information theory-based methods and cross-correlation to estimate the functional connectivity in cortical networks," PLoS ONE, vol. 4, no. 8, Article ID e6482, 2009.

[111] A. E. Pereda, "Electrical synapses and their functional interactions with chemical synapses," Nature Reviews Neuroscience, vol. 15, no. 4, pp. 250-263, 2014.

[112] M. Klein and E. R. Kandel, "Mechanism of calcium current modulation underlying presynaptic facilitation and behavioral sensitization in Aplysia," Proceedings of the National Academy of Sciences of the United States of America, vol. 77, no. 11 I, pp. 6912-6916, 1980.

[113] K. Delaney, D. W. Tank, and R. S. Zucker, "Presynaptic calcium and serotonin-mediated enhancement of transmitter release at crayfish neuromuscular junction," The Journal of Neuroscience, vol. 11, no. 9, pp. 2631-2643, 1991.

[114] P. S. Katz and W. N. Frost, "Intrinsic neuromodulation in the Tritonia swim CPG: the serotonergic dorsal swim interneurons act presynaptically to enhance transmitter release from interneuron $\mathrm{C}_{2}$," The Journal of Neuroscience, vol. 15, no. 9, pp. 6035-6045, 1995.

[115] P. G. Haydon, D. P. McCobb, and S. B. Kater, "Serotonin selectively inhibits growth cone motility and synaptogenesis of specific identified neurons," Science, vol. 226, no. 4674, pp. 561-564, 1984.

[116] P. G. Haydon and S. B. Kater, "The differential regulation of formation of chemical and electrical connections in Helisoma," Journal of Neurobiology, vol. 19, no. 7, pp. 636-655, 1988.

[117] R. D. Hadley, S. B. Kater, and C. S. Cohan, "Electrical synapse formation depends on interaction of mutually growing neurites," Science, vol. 221, no. 4609, pp. 466-468, 1983.

[118] R. D. Hadley, D. A. Bodnar, and S. B. Kater, "Formation of electrical synapses between isolated, cultured Helisoma neurons requires mutual neurite elongation," The Journal of Neuroscience, vol. 5, no. 12, pp. 3145-3153, 1985.

[119] R. D. Hadley and S. B. Kater, "Competence to form electrical connections is restricted to growing neurites in the snail, Helisoma," The Journal of Neuroscience, vol. 3, no. 5, pp. 924-932, 1983.

[120] P. G. Haydon, D. P. McCobb, and S. B. Kater, “The regulation of neurite outgrowth, growth cone mobility, and electrical synaptogenesis by serotonin," Journal of Neurobiology, vol. 18, no. 2, pp. 197-215, 1987.

[121] J. I. Goldberg, "Serotonin regulation of neurite outgrowth in identified neurons from mature and embryonic Helisoma trivolvis," Perspectives on Developmental Neurobiology, vol. 5, no. 4, pp. 373-387, 1998.

[122] R. C. Berdan, R. R. Shivers, and A. G. Bulloch, "Chemical synapses, particle arrays, pseudo-gap junctions and gap junctions of neurons and glia in the buccal ganglion of Helisoma," Synapse, vol. 1, no. 4, pp. 304-323, 1987.

[123] P. B. Guthrie, R. E. Lee, V. Rehder, M. F. Schmidt, and S. B. Kater, "Self-recognition: a constraint on the formation of electrical coupling in neurons," Journal of Neuroscience, vol. 14, no. 3, pp. 1477-1485, 1994.
[124] T. M. Szabo, D. S. Faber, and M. J. Zoran, "Transient electrical coupling delays the onset of chemical neurotransmission at developing synapses," The Journal of Neuroscience, vol. 24, no. 1, pp. 112-120, 2004.

[125] B. Rörig and B. Sutor, "Serotonin regulates gap junction coupling in the developing rat somatosensory cortex," European Journal of Neuroscience, vol. 8, no. 8, pp. 1685-1695, 1996.

[126] L. Sikich, J. M. Hickok, and R. D. Todd, "5-HT1A receptors control neurite branching during development," Developmental Brain Research, vol. 56, no. 2, pp. 269-274, 1990.

[127] J. J. Dudok, A. J. A. Groffen, M. P. Witter, P. Voorn, and M. Verhage, "Chronic activation of the $5-\mathrm{HT}_{2}$ receptor reduces 5HT neurite density as studied in organotypic slice cultures," Brain Research, vol. 1302, pp. 1-9, 2009.

[128] M. V. L. Bennett and R. S. Zukin, "Electrical coupling and neuronal synchronization in the mammalian brain," Neuron, vol. 41, no. 4, pp. 495-511, 2004.

[129] R. G. Kavasseri and R. Nagarajan, "Synchronization in electrically coupled neural networks," Complex Systems, vol. 16, no. 4, pp. 369-380, 2006.

[130] M. Ichikawa, K. Muramoto, K. Kobayashi, M. Kawahara, and Y. Kuroda, "Formation and maturation of synapses in primary cultures of rat cerebral cortical cells: an electron microscopic study," Neuroscience Research, vol. 16, no. 2, pp. 95-103, 1993.

[131] K. Muramoto, M. Ichikawa, M. Kawahara, K. Kobayashi, and Y. Kuroda, "Frequency of synchronous oscillations of neuronal activity increases during development and is correlated to the number of synapses in cultured cortical neuron networks," Neuroscience Letters, vol. 163, no. 2, pp. 163-165, 1993.

[132] G. Buzsáki and A. Draguhn, "Neuronal olscillations in cortical networks," Science, vol. 304, no. 5679, pp. 1926-1929, 2004.

[133] J. van Pelt, M. A. Corner, P. S. Wolters, W. L. C. Rutten, and G. J. A. Ramakers, "Long-term stability and developmental changes in spontaneous network burst firing patterns in dissociated rat cerebral cortex cell cultures on multi-electrode arrays," Neuroscience Letters, vol. 361, no. 1-3, pp. 86-89, 2004.

[134] M. Chiappalone, A. Vato, L. Berdondini, M. Koudelka-Hep, and S. Martinoia, "Network dynamics and synchronous activity in cultured cortical neurons," International Journal of Neural Systems, vol. 17, no. 2, pp. 87-103, 2007.

[135] A. Kapur and L. B. Haberly, "Duration of NMDA-dependent synaptic potentiation in piriform cortex in vivo is increased after epileptiform bursting," Journal of Neurophysiology, vol. 80, no. 4, pp. 1623-1629, 1998.

[136] D. Eytan and S. Marom, "Dynamics and effective topology underlying synchronization in networks of cortical neurons," Journal of Neuroscience, vol. 26, no. 33, pp. 8465-8476, 2006.

[137] E. Maeda, H. P. C. Robinson, and A. Kawana, “The mechanisms of generation and propagation of synchronized bursting in developing networks of cortical neurons," Journal of Neuroscience, vol. 15, no. 10, pp. 6834-6845, 1995.

[138] L. Zullo, M. Chiappalone, S. Martinoia, and F. Benfenati, "A 'spike-based' grammar underlies directional modification in network connectivity: effect on bursting activity and implications for bio-hybrids systems," PLoS ONE, vol. 7, no. 11, Article ID e49299, 2012.

[139] E. Maeda, Y. Kuroda, H. P. C. Robinson, and A. Kawana, "Modification of parallel activity elicited by propagating bursts in developing networks of rat cortical neurones," European Journal of Neuroscience, vol. 10, no. 2, pp. 488-496, 1998. 
[140] Y. Jimbo, H. P. C. Robinson, and A. Kawana, "Strengthening of synchronized activity by tetanic stimulation in cortical cultures: application of planar electrode," IEEE Transactions on Biomedical Engineering, vol. 45, no. 11, pp. 1297-1304, 1998.

[141] Y. Jimbo, T. Tateno, and H. P. C. Robinson, "Simultaneous induction of pathway-specific potentiation and depression in networks of cortical neurons," Biophysical Journal, vol. 76, no. 2, pp. 670-678, 1999.

[142] T. Tateno and Y. Jimbo, "Activity-dependent enhancement in the reliability of correlated spike timings in cultured cortical neurons," Biological Cybernetics, vol. 80, no. 1, pp. 45-55, 1999.

[143] G. Shahaf and S. Marom, "Learning in networks of cortical neurons," The Journal of Neuroscience, vol. 21, no. 22, pp. 8782-8788, 2001.

[144] M. E. Ruaro, P. Bonifazi, and V. Torre, "Toward the neurocomputer: image processing and pattern recognition with neuronal cultures," IEEE Transactions on Biomedical Engineering, vol. 52, no. 3, pp. 371-383, 2005.

[145] D. A. Wagenaar, J. Pine, and S. M. Potter, "Searching for plasticity in dissociated cortical cultures on multi-electrode arrays," Journal of Negative Results in BioMedicine, vol. 5, article 16, 2006.

[146] J. Le Feber, J. Stegenga, and W. L. C. Rutten, “The effect of slow electrical stimuli to achieve learning in cultured networks of rat cortical neurons," PLoS ONE, vol. 5, no. 1, Article ID e8871, 2010.

[147] G. W. Van Staveren, J. R. Buitenweg, E. Marani, and W. L. C. Rutten, "The effect of training of cultured neuronal networks: can they learn ?" in Proceedings of the 2nd International IEEE EMBS Conference on Neural Engineering, pp. 328-331, Arlington, Va, USA, March 2005.

[148] C. P. Marder and D. V. Buonomano, "Timing and balance of inhibition enhance the effect of long-term potentiation on cell firing," The Journal of Neuroscience, vol. 24, no. 40, pp. 88738884, 2004.

[149] G.-Q. Bi and M.-M. Poo, "Distributed synaptic modification in neural networks induced by patterned stimulation," Nature, vol. 401, no. 21, pp. 792-796, 1999.

[150] A. B. Mulder, M. P. M. Arts, and F. H. Lopes da Silva, "Short- and long-term plasticity of the hippocampus to nucleus accumbens and prefrontal cortex pathways in the rat, in vivo," European Journal of Neuroscience, vol. 9, no. 8, pp. 1603-1611, 1997.

[151] C. M. Werk and C. A. Chapman, "Long-term potentiation of polysynaptic responses in layer $\mathrm{V}$ of sensorimotor cortex induced by theta-patterned tetanization in the awake rat," Cerebral Cortex, vol. 13, no. 5, pp. 500-507, 2003.

[152] O. Paulsen and T. J. Sejnowski, "Natural patterns of activity and long-term synaptic plasticity," Current Opinion in Neurobiology, vol. 10, no. 2, pp. 172-180, 2000.

[153] P. K. Stanton and T. J. Sejnowski, "Associative long-term depression in the hippocampus induced by hebbian covariance," Nature, vol. 339, no. 6221, pp. 215-218, 1989.

[154] Y.-Y. Huang, C. Pittenger, and E. R. Kandel, "A form of longlasting, learning-related synaptic plasticity in the hippocampus induced by heterosynaptic low-frequency pairing," Proceedings of the National Academy of Sciences of the United States of America, vol. 101, no. 3, pp. 859-864, 2004.

[155] Z. C. Chao, D. J. Bakkum, and S. M. Potter, "Region-specific network plasticity in simulated and living cortical networks: comparison of the center of activity trajectory (CAT) with other statistics," Journal of Neural Engineering, vol. 4, no. 3, pp. 294308, 2007.
[156] R. Madhavan, Z. C. Chao, and S. M. Potter, "Plasticity of recurring spatiotemporal activity patterns in cortical networks," Physical Biology, vol. 4, no. 3, pp. 181-193, 2007.

[157] R. L. Berry, T. J. Teyler, and H. Taizhen, "Induction of LTP in rat primary visual cortex: tetanus parameters," Brain Research, vol. 481, no. 2, pp. 221-227, 1989.

[158] Y. Dan and M.-M. Poo, "Spike timing-dependent plasticity of neural circuits," Neuron, vol. 44, no. 1, pp. 23-30, 2004.

[159] M. A. Corner, J. van Pelt, P. S. Wolters, R. E. Baker, and R. H. Nuytinck, "Physiological effects of sustained blockade of excitatory synaptic transmission on spontaneously active developing neuronal networks-an inquiry into the reciprocal linkage between intrinsic biorhythms and neuroplasticity in early ontogeny," Neuroscience and Biobehavioral Reviews, vol. 26, no. 2, pp. 127-185, 2002.

[160] Q. Zhou and M.-M. Poo, "Reversal and consolidation of activity-induced synaptic modifications," Trends in Neurosciences, vol. 27, no. 7, pp. 378-383, 2004.

[161] A. Pimashkin, A. Gladkov, I. Mukhina, and V. Kazantsev, "Adaptive enhancement of learning protocol in hippocampal cultured networks grown on multielectrode arrays," Frontiers in Neural Circuits, vol. 7, article 87, 2013.

[162] Z. C. Chao, D. J. Bakkum, and S. M. Potter, "Shaping embodied neural networks for adaptive goal-directed behavior," PLoS Computational Biology, vol. 4, no. 3, Article ID e1000042, 17 pages, 2008.

[163] S. Martinoia, V. Sanguineti, L. Cozzi et al., "Towards an embodied in-vitro electrophysiology: the NeuroBIT project," Neurocomputing, vol. 58-60, pp. 1065-1072, 2004.

[164] A. Novellino, P. D’Angelo, L. Cozzi, M. Chiappalone, V. Sanguineti, and S. Martinoia, "Connecting neurons to a mobile robot: an in vitro bidirectional neural interface," Computational Intelligence and Neuroscience, vol. 2007, Article ID 12725, 13 pages, 2007.

[165] K. Warwick, D. Xydas, S. J. Nasuto et al., "Controlling a mobile robot with a biological brain," Defence Science Journal, vol. 60, no. 1, pp. 5-14, 2010.

[166] J. Tessadori, M. Bisio, S. Martinoia, and M. Chiappalone, "Modular neuronal assemblies embodied in a closed-loop environment: towards future integration of brains and machines," Frontiers in Neural Circuits, vol. 6, article 99, 2012.

[167] A. Wallach, D. Eytan, A. Gal, C. Zrenner, and S. Marom, "Neuronal response clamp," Frontiers in Neuroengineering, vol. 4, article 3, 2011.

[168] A. Wallach and S. Marom, "Interactions between network synchrony and the dynamics of neuronal threshold," Journal of Neurophysiology, vol. 107, no. 11, pp. 2926-2936, 2012.

[169] H. Keren and S. Marom, "Controlling neural network responsiveness: tradeoffs and constraints," Frontiers in Neuroengineering, vol. 7, article 11, 2014.

[170] M. Frega, M. Tedesco, P. Massobrio, M. Pesce, and S. Martinoia, "Network dynamics of 3D engineered neuronal cultures: a new experimental model for in-vitro electrophysiology," Scientific Reports, vol. 4, article 5489, 2014.

[171] T. T. Kanagasabapathi, P. Massobrio, R. A. Barone et al., "Functional connectivity and dynamics of cortical-thalamic networks co-cultured in a dual compartment device," Journal of Neural Engineering, vol. 9, no. 3, Article ID 036010, 2012.

[172] P. Massobrio, L. de Arcangelis, V. Pasquale, H. J. Jensen, and D. Plenz, "Criticality as a signature of healthy neural systems," Frontiers in Systems Neuroscience, vol. 9, article 22, 2015. 
[173] H. A. Johnson, A. Goel, and D. V. Buonomano, "Neural dynamics of in vitro cortical networks reflects experienced temporal patterns," Nature Neuroscience, vol. 13, no. 8, pp. 917-919, 2010.

[174] A. Borst and F. E. Theunissen, "Information theory and neural coding," Nature Neuroscience, vol. 2, no. 11, pp. 947-957, 1999. 

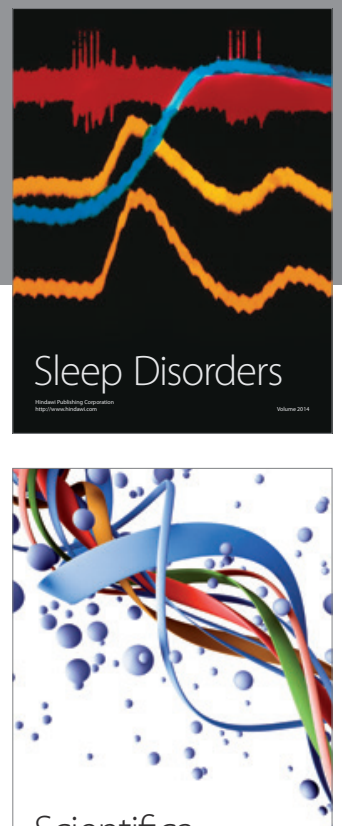

Scientifica
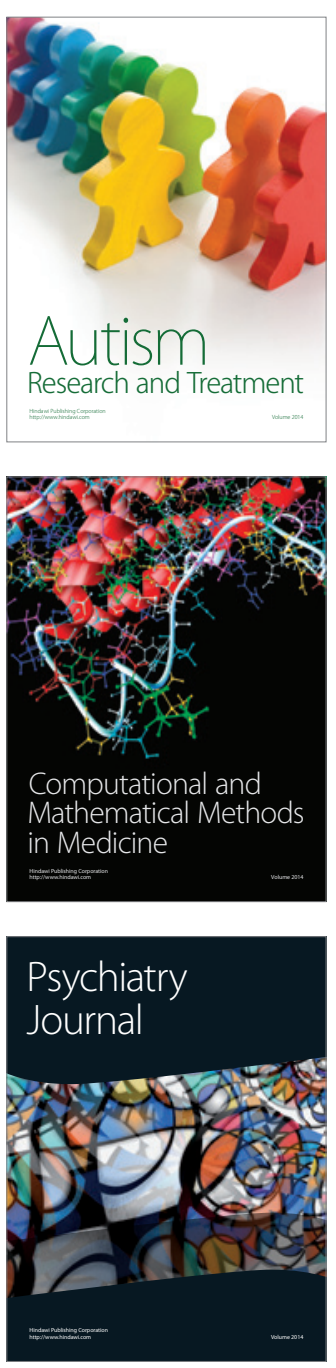
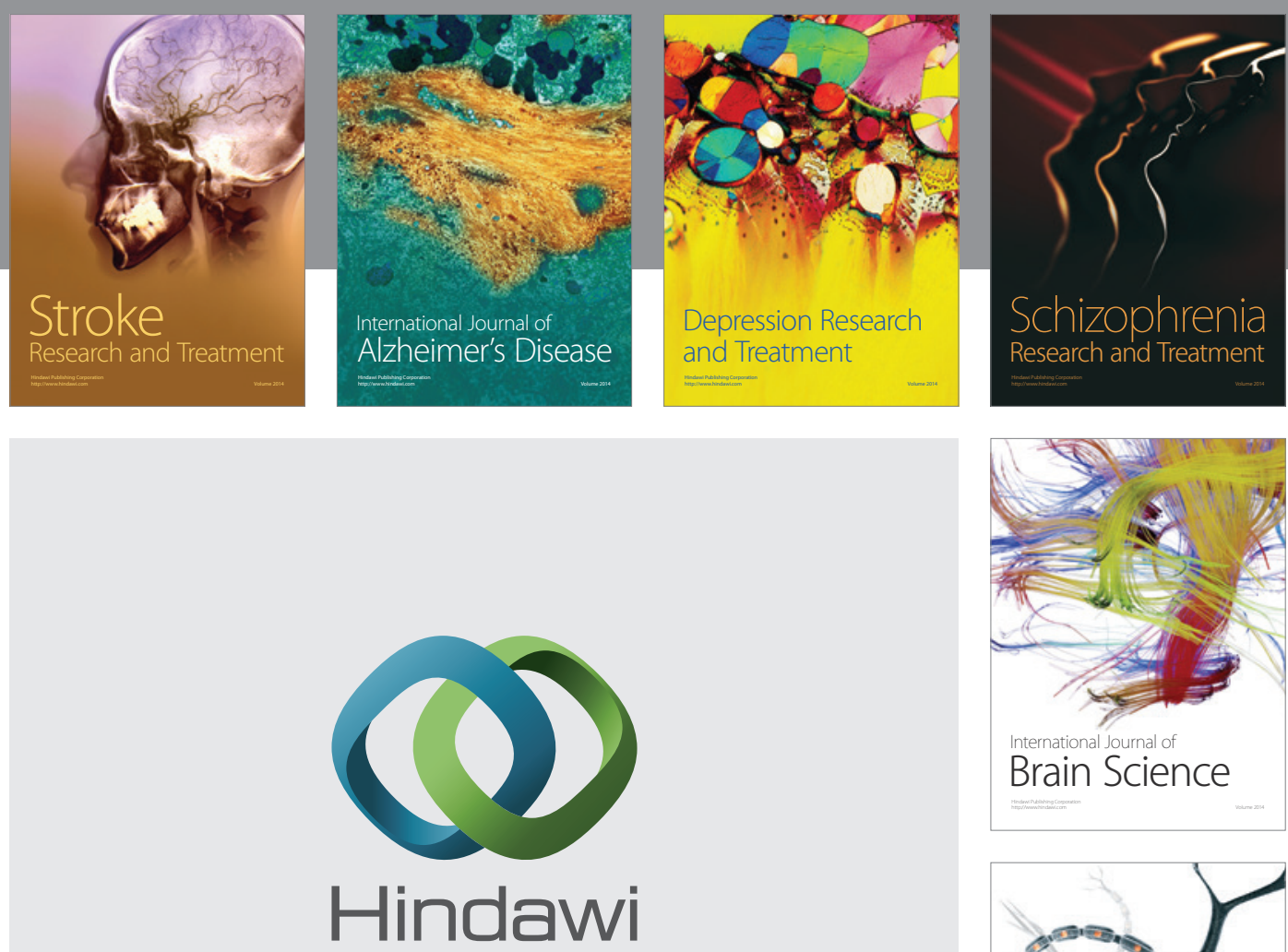

Submit your manuscripts at

http://www.hindawi.com
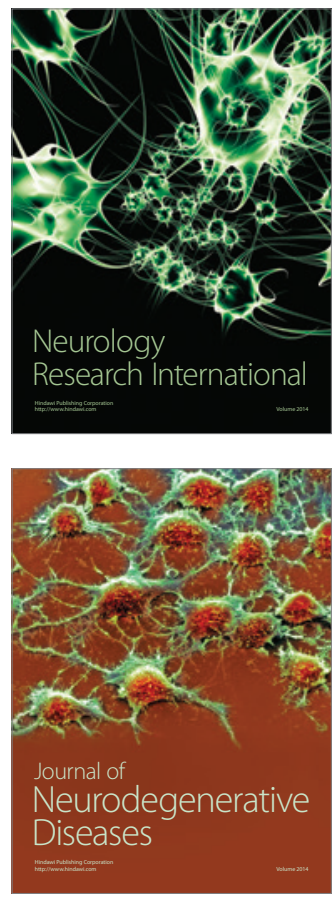

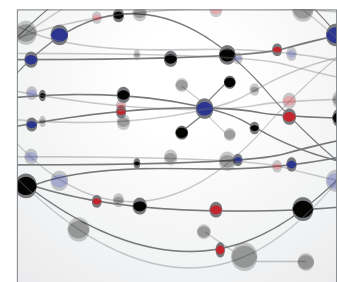

The Scientific World Journal
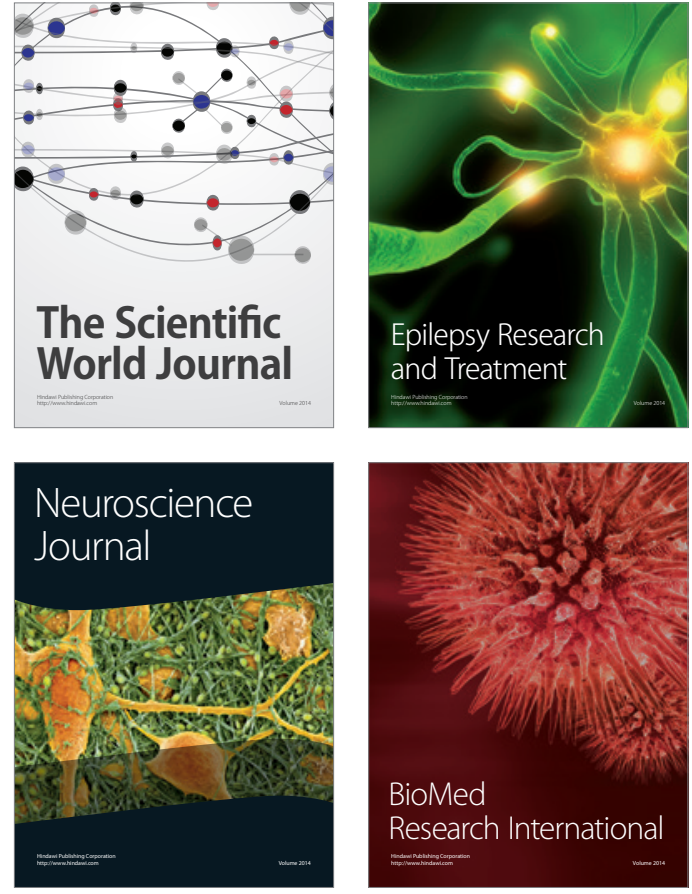

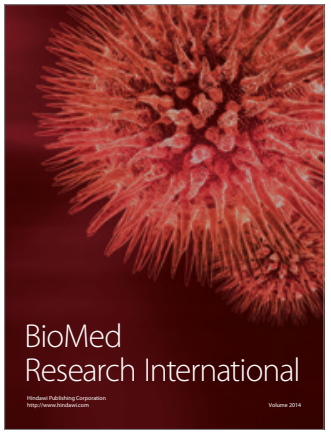

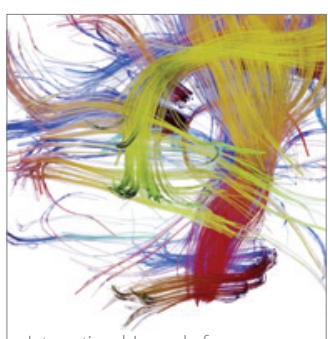

Brain Science

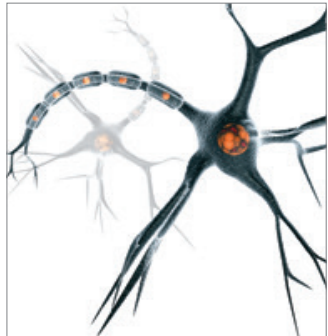

Neural Plasticity
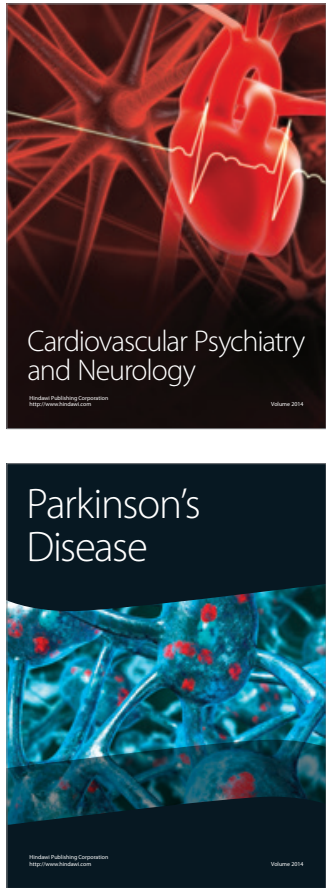